\title{
Ring-Chain Tautomerism in Organic Synthesis: Synthesis of Heterocyclic Enamines from a Novel and Practical Formal Ring Transformation Reaction of Lactones
}

Mei-Xiang Wang, ${ }^{a} *$ Yong Liu, ${ }^{a}$ Hong-Yun Gao, ${ }^{a}$ Yan Zhang, ${ }^{a}$ Chu-Yi Yu, ${ }^{a}$ Zhi-Tang Huang $^{a}$ and George W. J. Fleet ${ }^{b}$

${ }^{a}$ Laboratory of Chemical Biology, Center for Molecular Science, Institute of Chemistry, Chinese Academy of Sciences, Beijing 100080, China. ${ }^{\mathrm{b}}$ Dyson Perrins Laboratory, Department of Chemistry, Oxford University, South Parks Road, Oxford, UK

mxwang@mail.iccas.ac.cn

Table of Contents

1. Spectral data of compounds 3

2. ${ }^{1} \mathrm{H}$ NMR spectra of $\mathbf{2}$ and $\mathbf{3},{ }^{1} \mathrm{H}$ NMR and ${ }^{13} \mathrm{C}$ NMR spectra of $\mathbf{6 a}, \mathbf{6 d}, \mathbf{6 g}, \mathbf{6 j}, \mathbf{6 m}$, $60,6 s, 6 x, 6 y$.

1. Spectral data of compounds 3 
tert-Butyl 6-methanesulfonyloxy-3-oxohexanoate (3a). Colorless oil: ${ }^{1} \mathrm{H}$ NMR $\left(\mathrm{CDCl}_{3}\right) \cdot 4.27(\mathrm{t}, J=6.1,2 \mathrm{H}), 3.38(\mathrm{~s}, 2 \mathrm{H}), 3.01(\mathrm{~s}, 3 \mathrm{H}), 2.73(\mathrm{t}, J=6.8,2 \mathrm{H}), 2.06$ (quin, $J=6.4,2 \mathrm{H}), 1.48(\mathrm{~s}, 9 \mathrm{H})($ A trace amount of enol tautomer was observed from ${ }^{1} \mathrm{H}$ NMR spectra); ${ }^{13} \mathrm{C}$ NMR $\left(\mathrm{CDCl}_{3}\right) \quad 201.9,166.1,81.9,68.6,50.2,38.0,37.0,27.7$, 22.7; IR (KBr) 1734, 1714, 1354, $1172 \mathrm{~cm}^{-1}$; MS (EI) m/z 207 (M-73, 3\%), 165 (7), $57(100)$

tert-Butyl 6-methanesulfonyloxy-4-methoxy-3-oxohexanoate (3b). Pale yellow oil: ${ }^{1} \mathrm{H}$ NMR $\left(\mathrm{CDCl}_{3}\right) \cdot 12.10(\mathrm{~s}, 0.25 \mathrm{H}, \mathrm{OH}), 5.12(\mathrm{~s}, 0.25 \mathrm{H},=\mathrm{CH}), 4.35-4.20(\mathrm{~m}$, 2H), 3.85-3.78 (m, 0.75H), 3.69-3.62 (m, 0.25H), $3.45(\mathrm{~s}, 1.5 \mathrm{H}), 3.39(\mathrm{~s}, 2.25 \mathrm{H}), 3.33$ (s, 0.75H), $2.98(\mathrm{~s}, 3 \mathrm{H}), 2.19-2.05(\mathrm{~m}, 2 \mathrm{H}), 1.46(\mathrm{~s}, 2.25 \mathrm{H}), 1.42(\mathrm{~s}, 6.75 \mathrm{H}) ; \mathrm{IR}(\mathrm{KBr})$ $1738,1718,1356,1174 \mathrm{~cm}^{-1}$.

tert-Butyl 4-alloxy-6-methanesulfonyloxy-3-oxohexanoate (3c). Colorless oil: ${ }^{1} \mathrm{H}$ $\mathrm{NMR}\left(\mathrm{CDCl}_{3}\right) \quad 12.2(\mathrm{~s}, 0.2 \mathrm{H}, \mathrm{OH}), 5.98-5.82(\mathrm{~m}, 1 \mathrm{H}), 5.34-5.19(\mathrm{~m}, 1.8 \mathrm{H}$, $\left.-\mathrm{CH}=\mathrm{CH}_{2}\right), 5.18(\mathrm{~s}, 1 \mathrm{H},=\mathrm{CH}), 4.43-4.28(\mathrm{~m}, 2 \mathrm{H}), 4.14-4.07(\mathrm{~m}, 1 \mathrm{H}), 4.05-3.99(\mathrm{~m}$, 1.6H), 3.93-3.86 (m, 0.4H), $3.51(\mathrm{~s}, 2 \mathrm{H}), 3.02(\mathrm{~s}, 2.4 \mathrm{H}), 3.01(\mathrm{~s}, 0.6 \mathrm{H}), 2.21-2.02(\mathrm{~m}$, 2H), 1.49 (s, 1.8H), 1.46 (s, 7.2H); IR (KBr) 1739, 1719, 1356, $1174 \mathrm{~cm}^{-1}$.

tert-Butyl 4-benzoxy-6-methanesulfonyloxy-3-oxohexanoate (3d). Colorless oil: ${ }^{1} \mathrm{H} \mathrm{NMR}\left(\mathrm{CDCl}_{3}\right) \quad 12.21(\mathrm{~s}, 0.2 \mathrm{H}, \mathrm{OH}), 7.34-7.25(\mathrm{~m}, 5 \mathrm{H}), 5.21(\mathrm{~s}, 0.2 \mathrm{H},=\mathrm{CH}), 4.90$ $(\mathrm{d}, J=11.3,0.2 \mathrm{H}), 4.68(\mathrm{~d}, J=11.3,0.2 \mathrm{H}), 4.60(\mathrm{~d}, J=11.3,0.8 \mathrm{H}), 4.49(\mathrm{~d}, J=11.3$ 0.8H), 4.40-4.20 (m, 2H), 4.08-4.03 (m, 0.8H), 3.98-3.93 (m, 0.2H), $3.48(\mathrm{~s}, 1.6 \mathrm{H})$, $2.90(\mathrm{~s}, 2.4 \mathrm{H}), 2.86(\mathrm{~s}, 0.6 \mathrm{H}), 2.20-2.00(\mathrm{~m}, 2 \mathrm{H}), 1.48(\mathrm{~s}, 1.8 \mathrm{H}), 1.41(\mathrm{~s}, 7.2 \mathrm{H})$. IR (KBr) $1738,1719,1356,1175 \mathrm{~cm}^{-1}$. 
4.3.5. tert-Butyl 7-methanesulfonyloxy-3-oxoheptanoate (3e). Colorless liquid: ${ }^{1} \mathrm{H}$ NMR $\left(\mathrm{CDCl}_{3}\right) \quad 4.24(\mathrm{t}, J=5.8,2 \mathrm{H}), 3.36(\mathrm{~s}, 2 \mathrm{H}), 3.02(\mathrm{~s}, 3 \mathrm{H}), 2.61(\mathrm{t}, J=6.7$, 2H), 1.76 (quin, $J=6.4,2 \mathrm{H}), 1.47(\mathrm{~s}, 9 \mathrm{H})$ (A trace amount of enol tautomer was observed from ${ }^{1} \mathrm{H}$ NMR spectra); IR (KBr) 1739, 1713, 1353, $1174 \mathrm{~cm}^{-1}$; MS (EI) $\mathrm{m} / \mathrm{z}$ $221(\mathrm{M}-73,6 \%), 179(22), 57$ (100).

2. ${ }^{1} \mathrm{H}$ NMR spectra of 2 and $3,{ }^{1} \mathrm{H}$ NMR and ${ }^{13} \mathrm{C}$ NMR spectra of $6 \mathrm{a}, 6 \mathrm{~d}, 6 \mathrm{~g}, 6 \mathrm{j}$, 6m, 6o, 6s, 6x, 6y. 

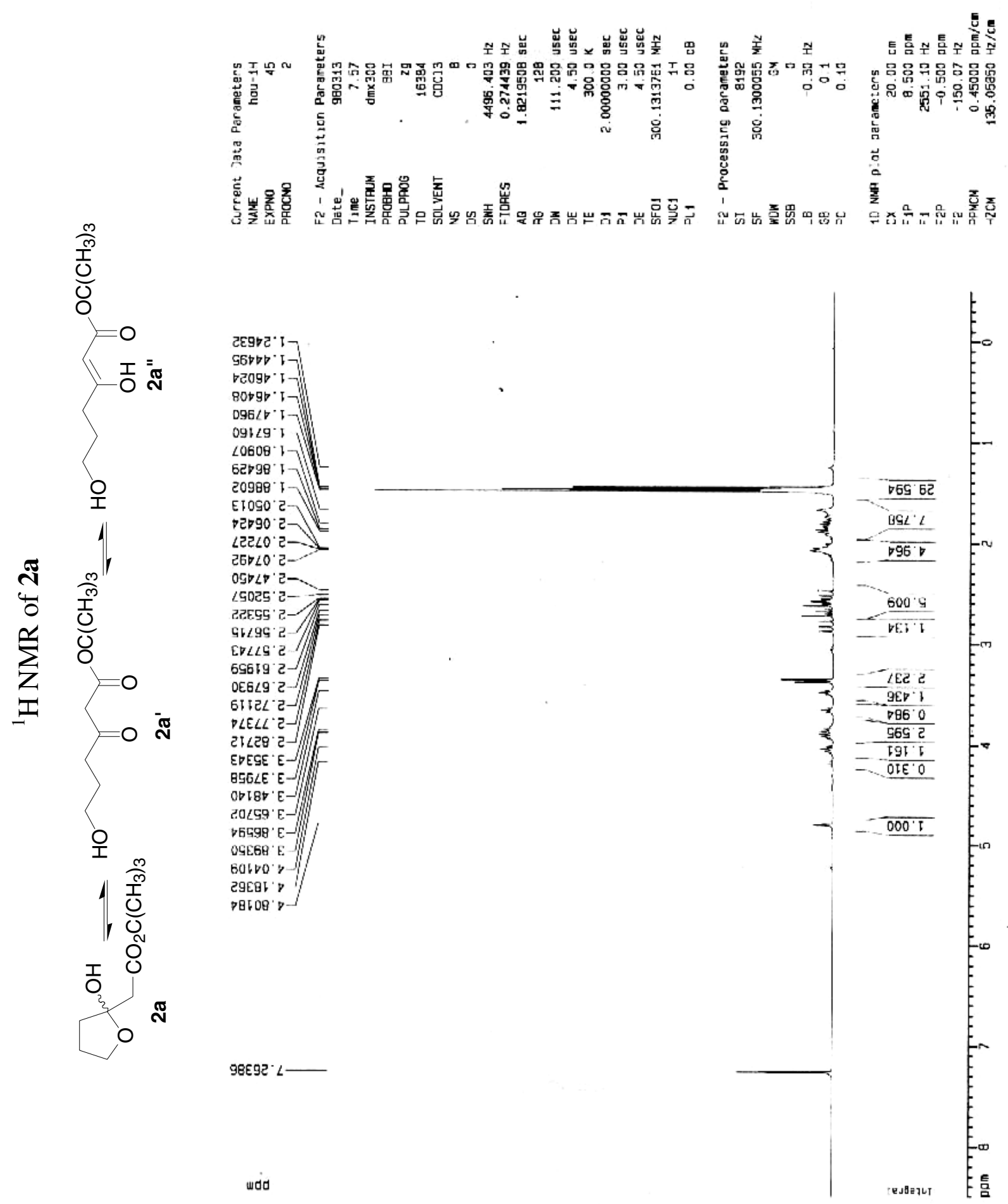


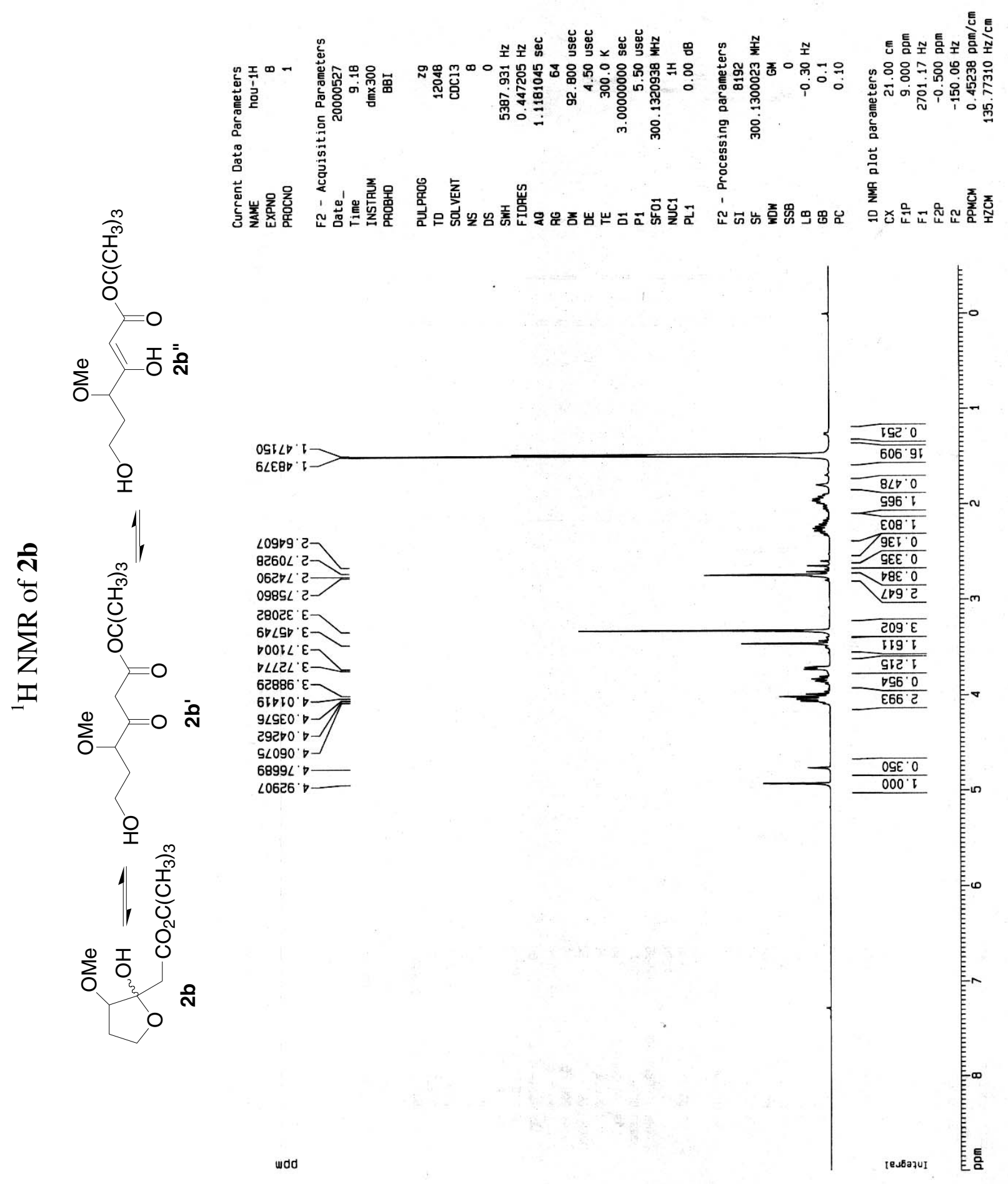



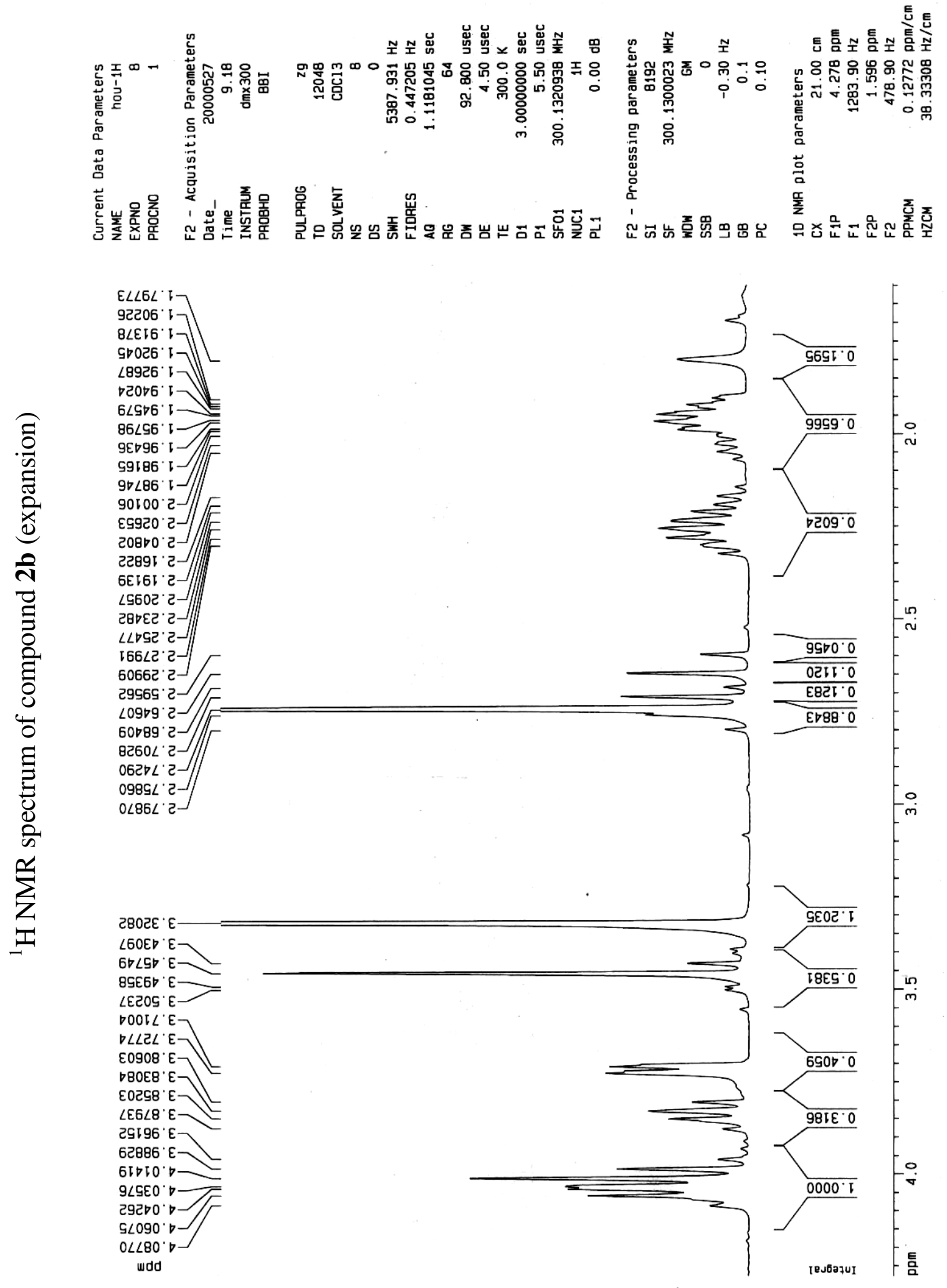


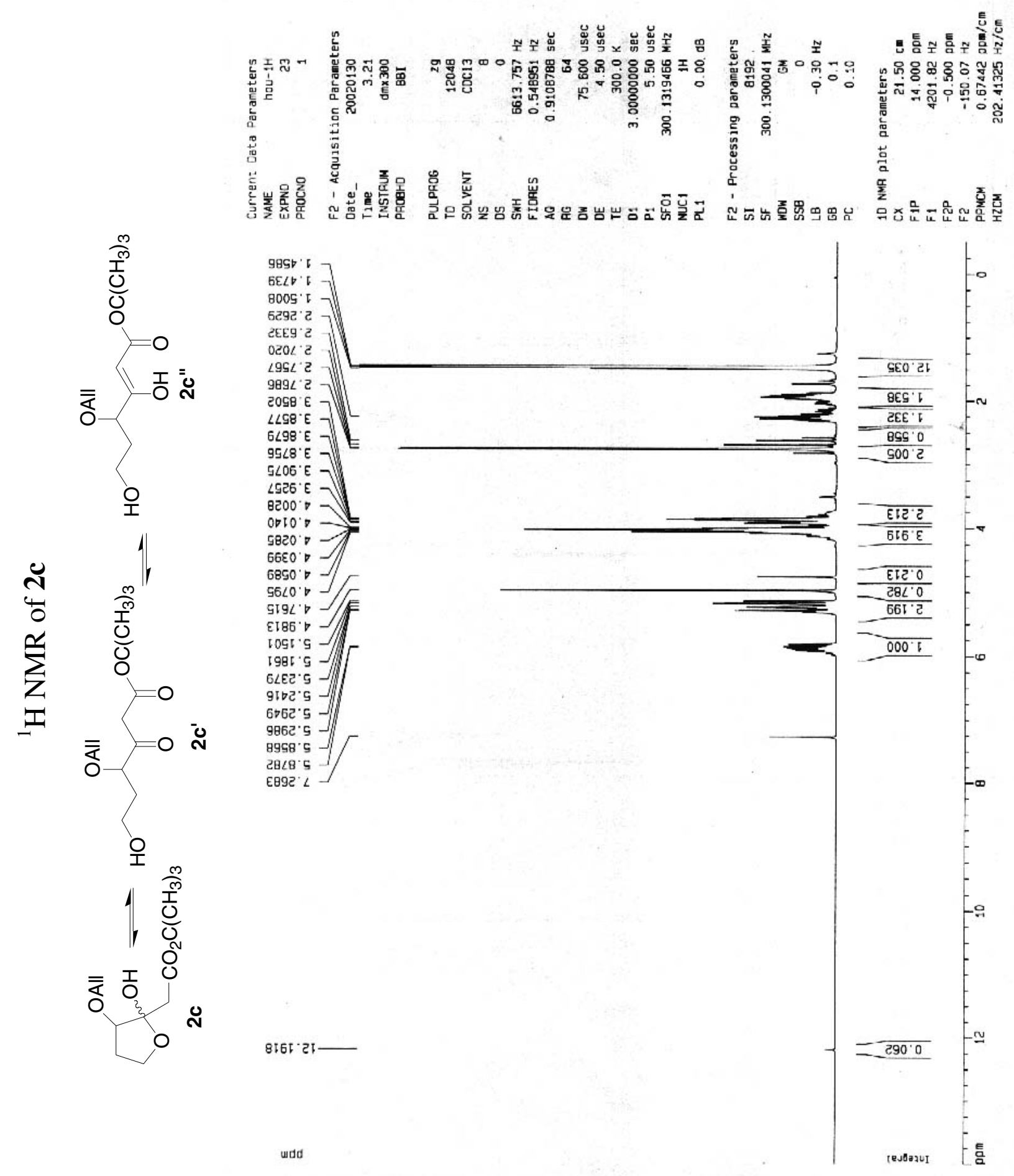



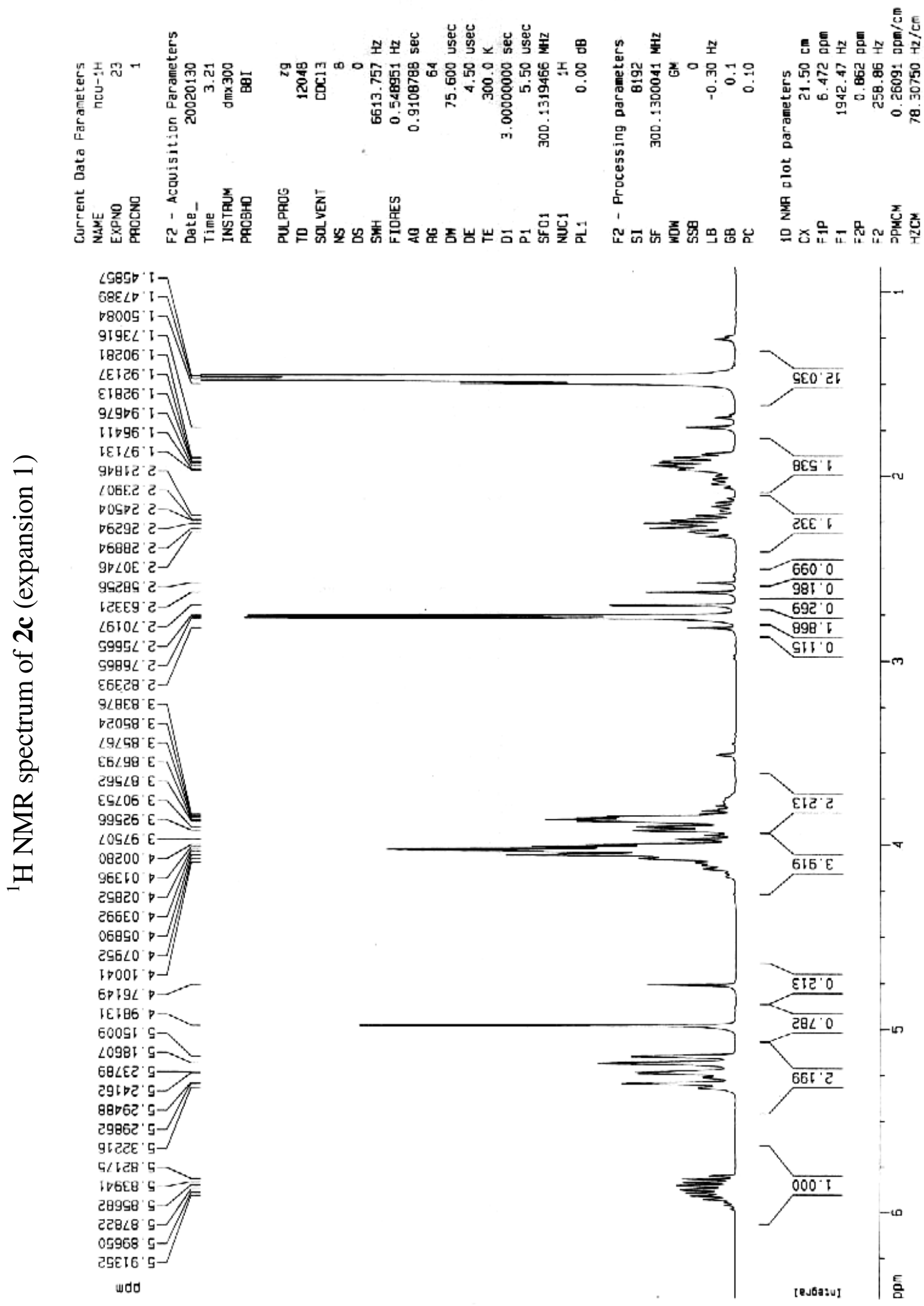


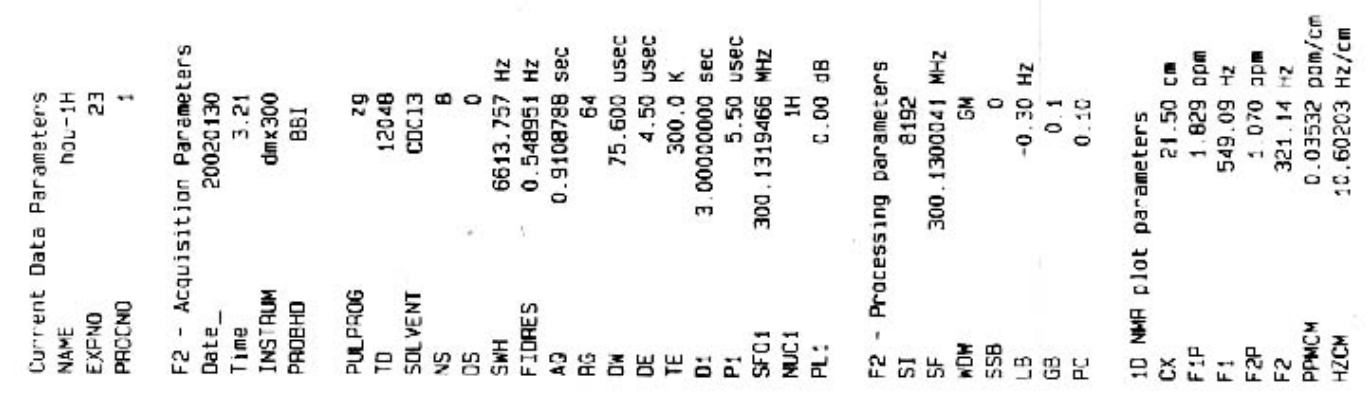

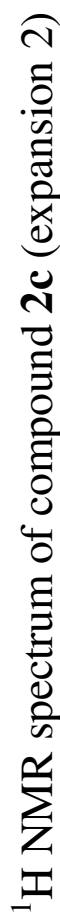

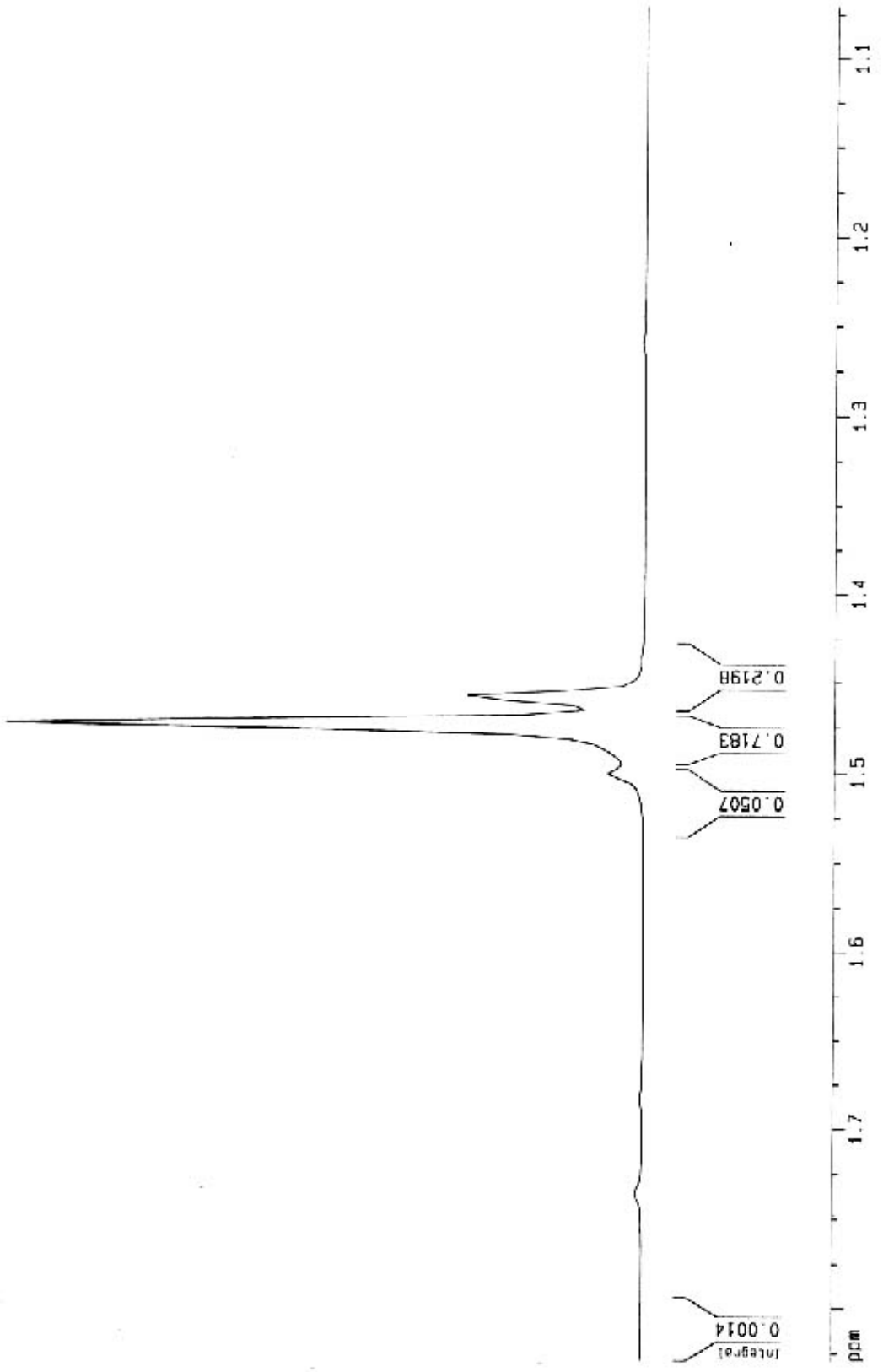



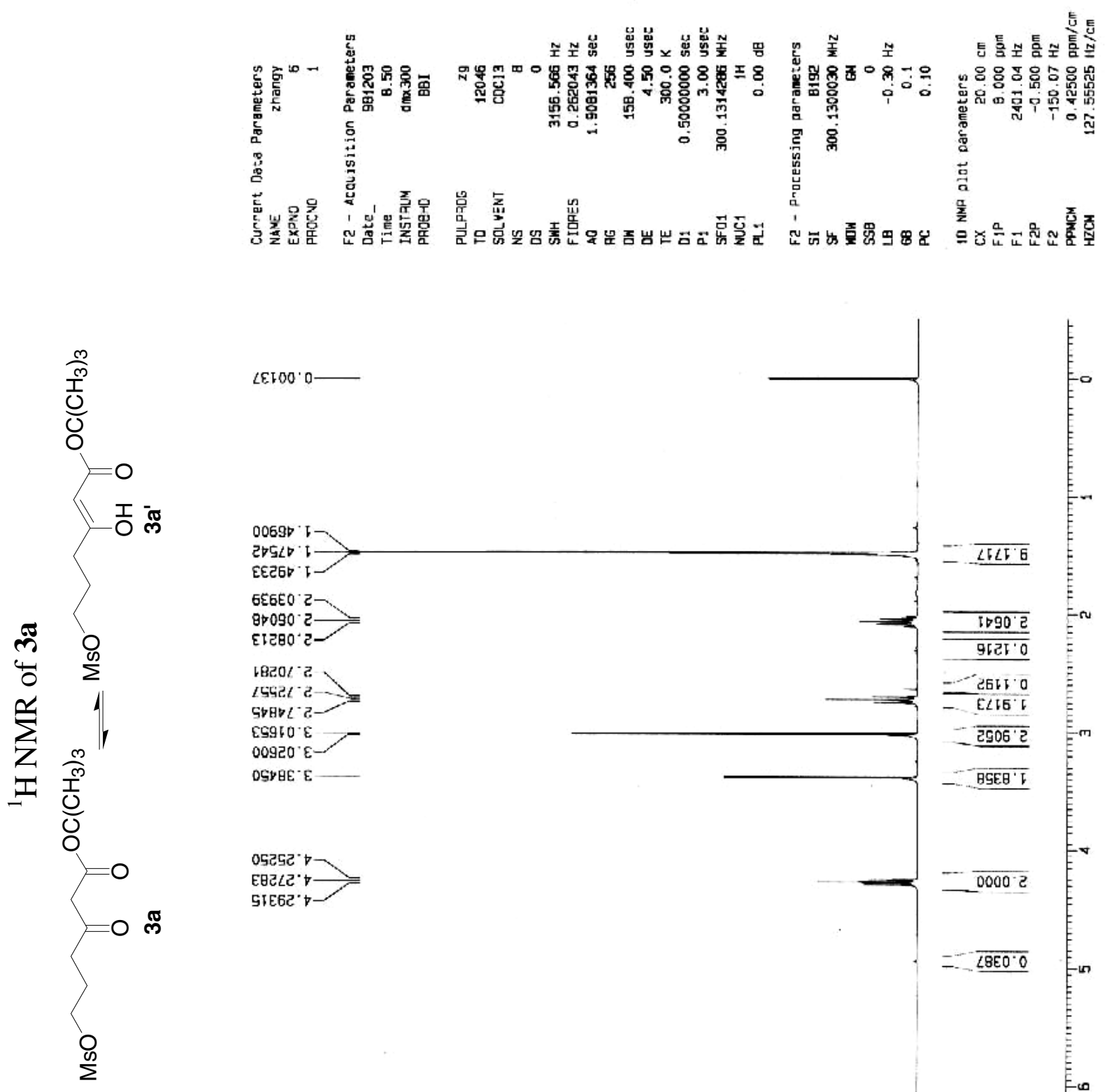

6E6EO $2-$

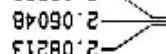

เB20 $/ 2$

1 sict $t^{\circ} \mathrm{C}-2$

ESgLO

$00950.8-$

DSPEE $E$

0525ट $\nabla$

E日ट $\angle C^{\circ} \downarrow$

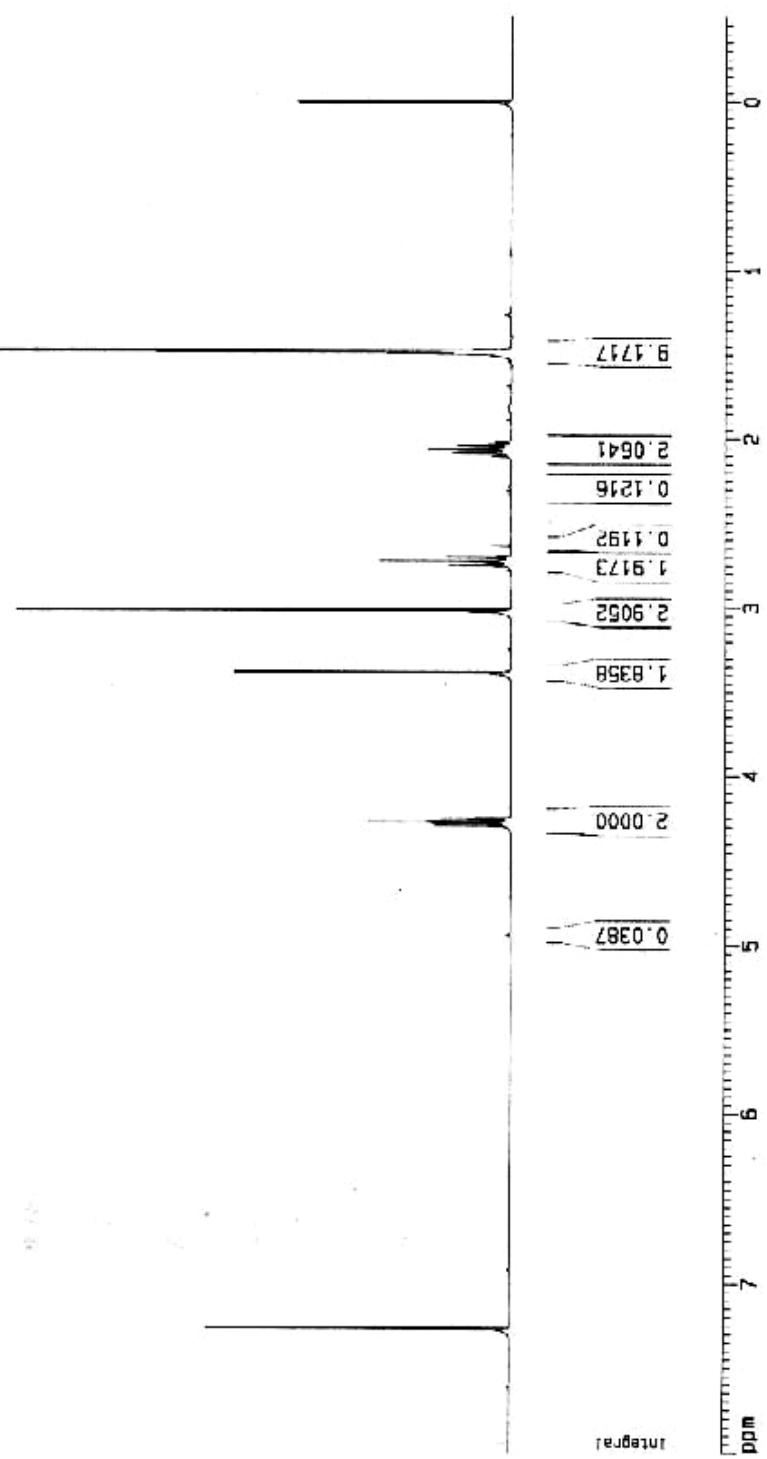

8D $\angle 2^{\circ} L-$

wdo 


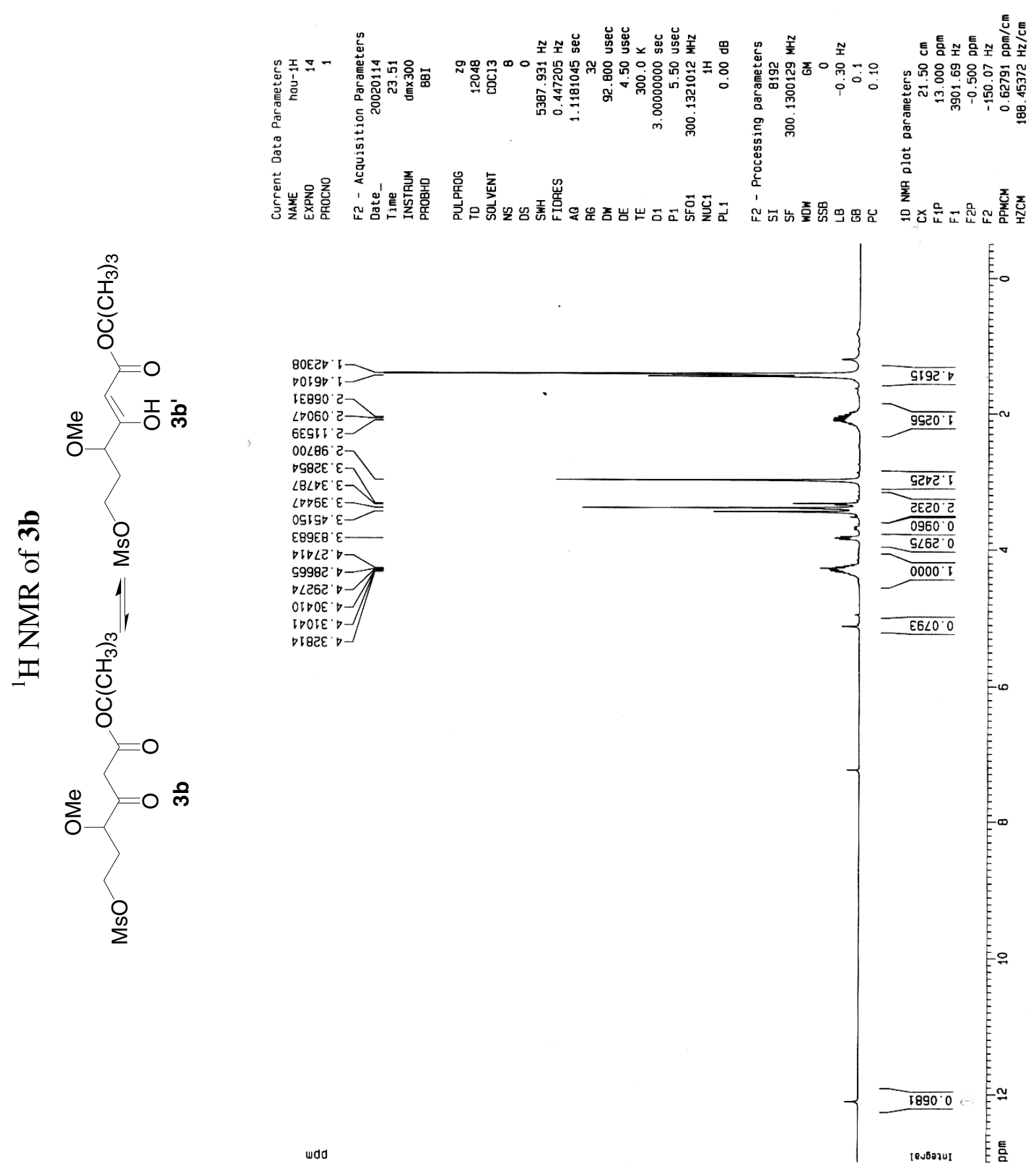




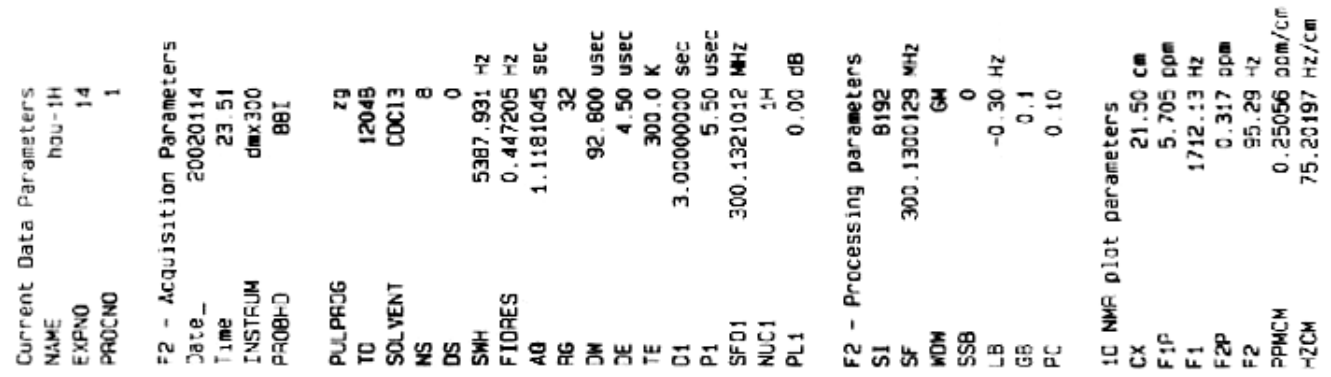

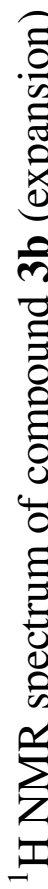

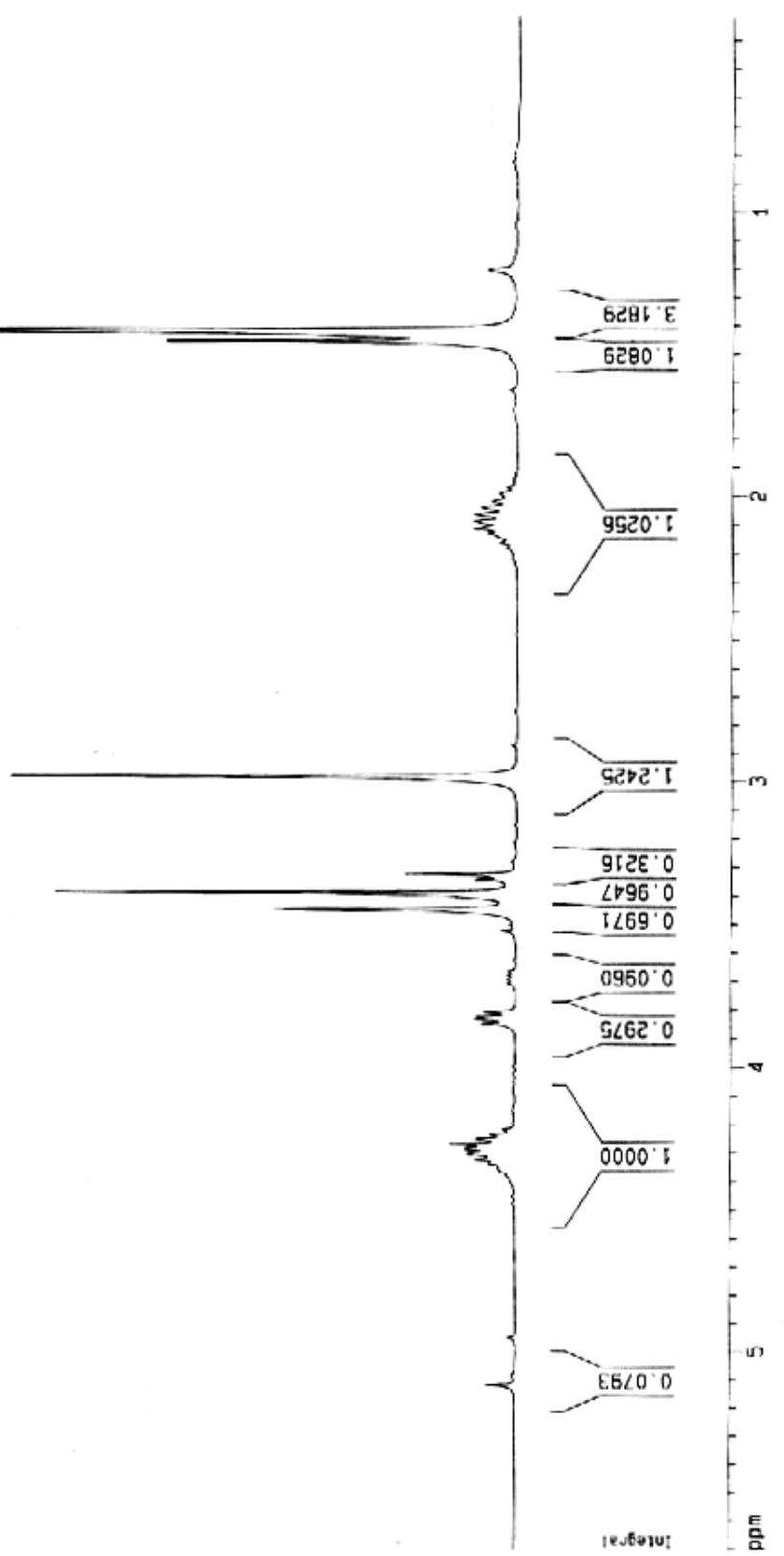



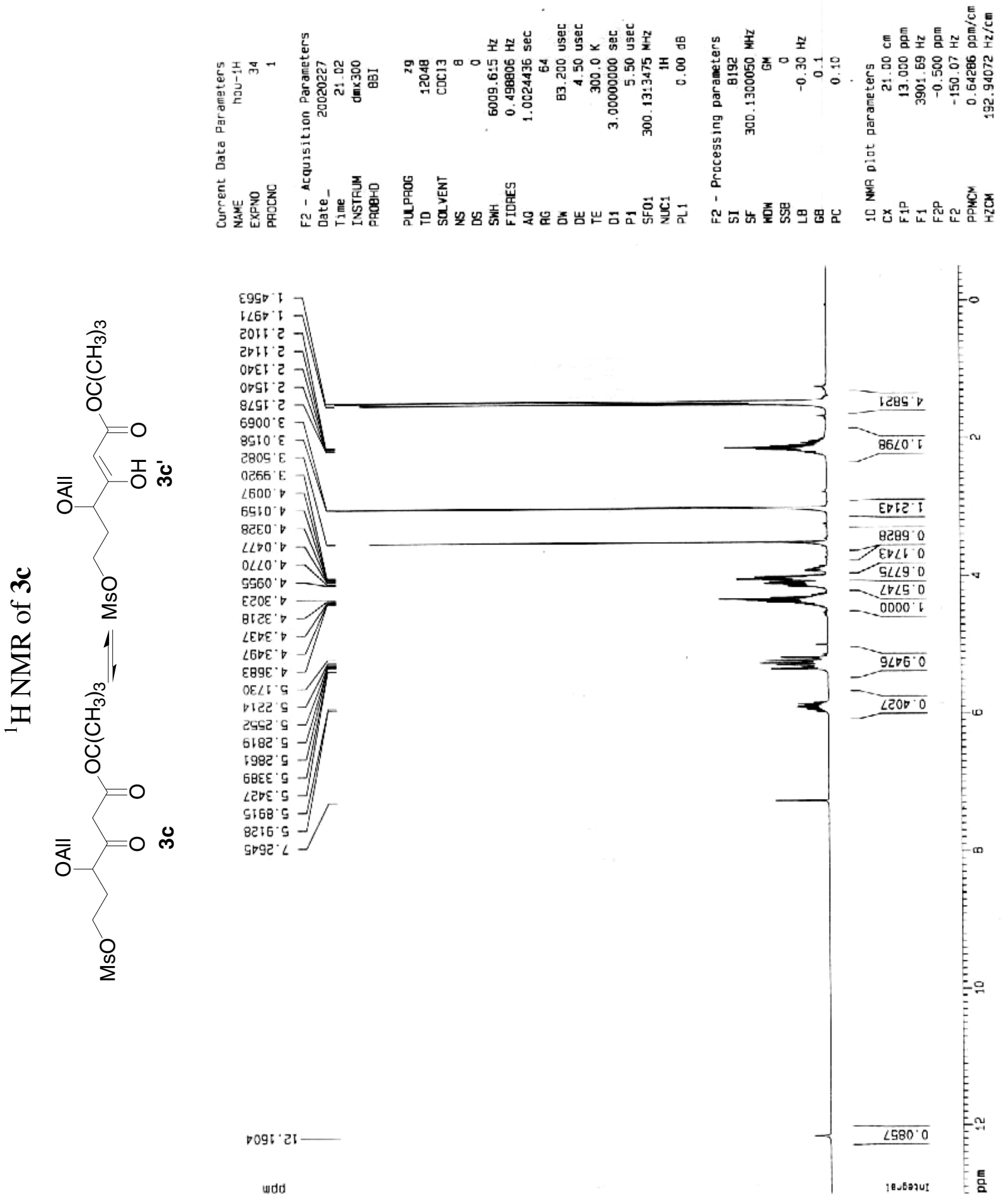

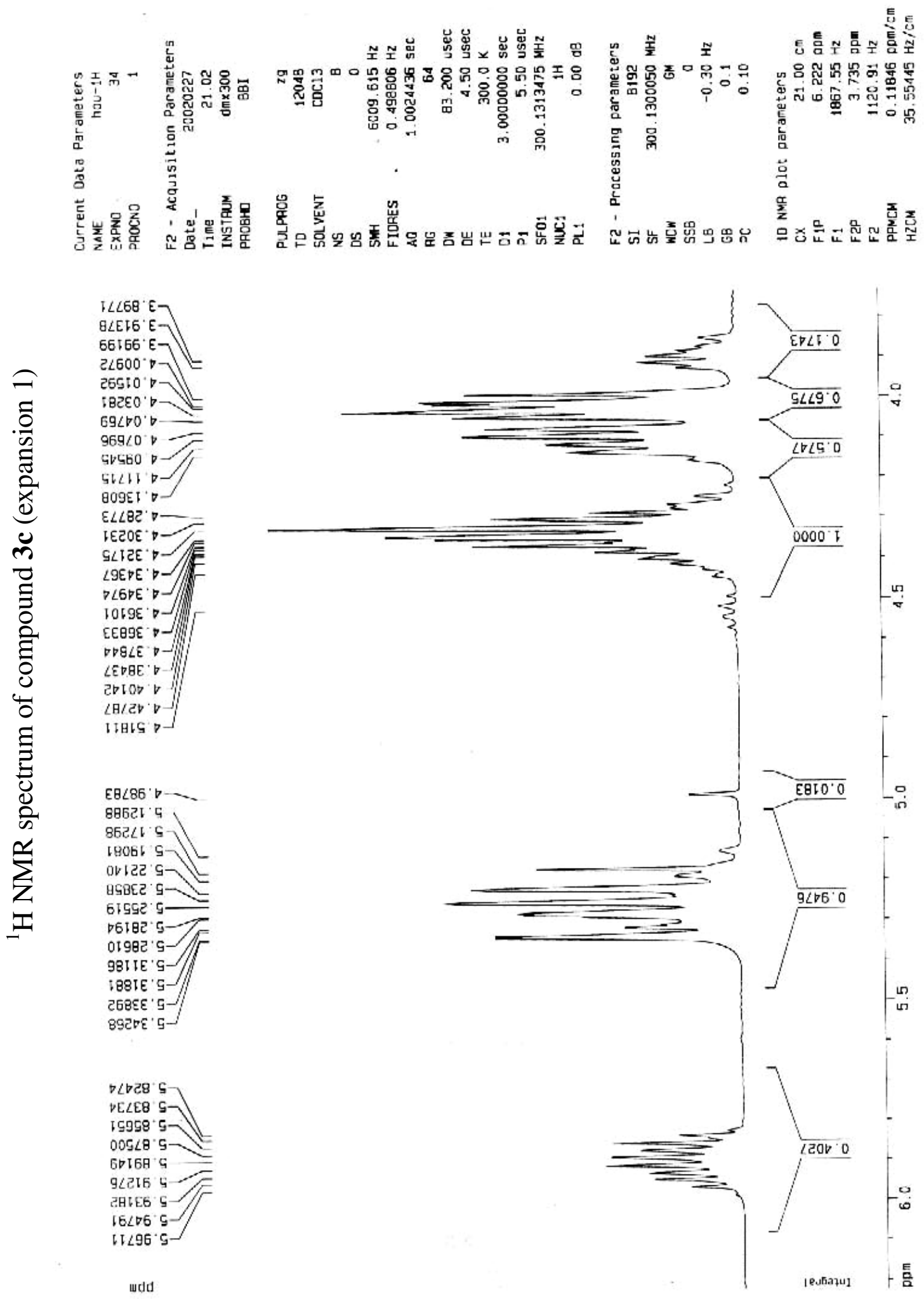


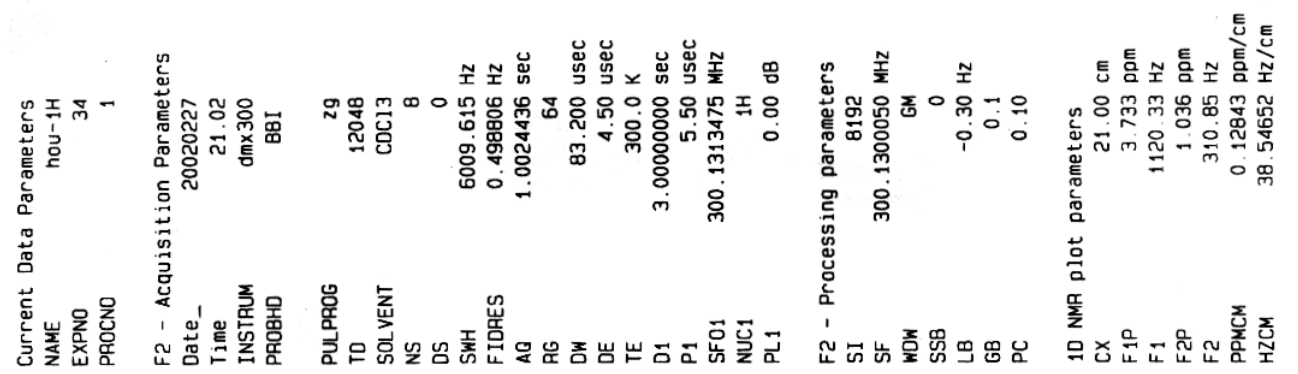

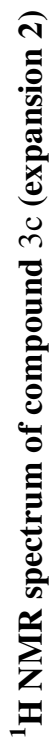

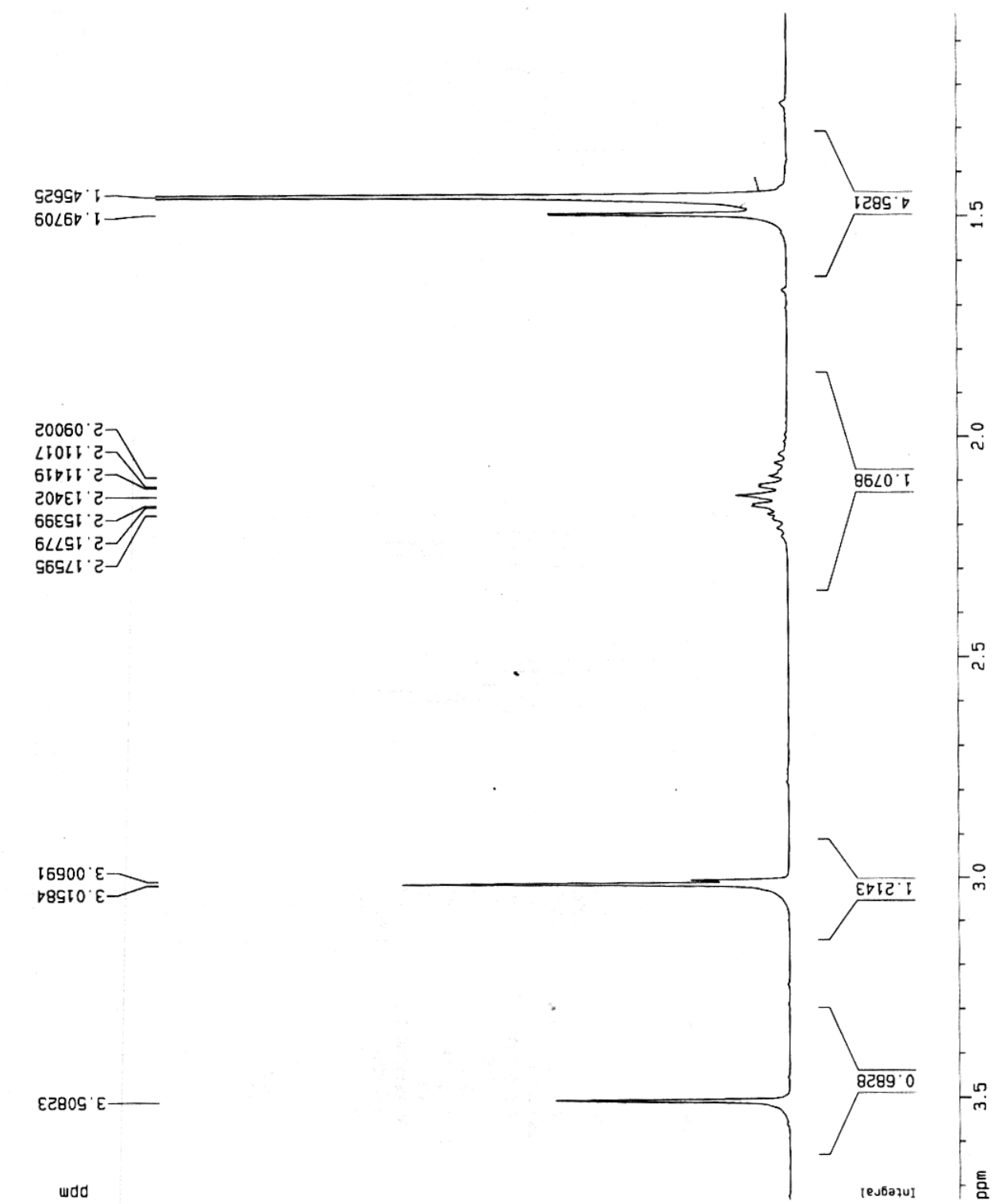



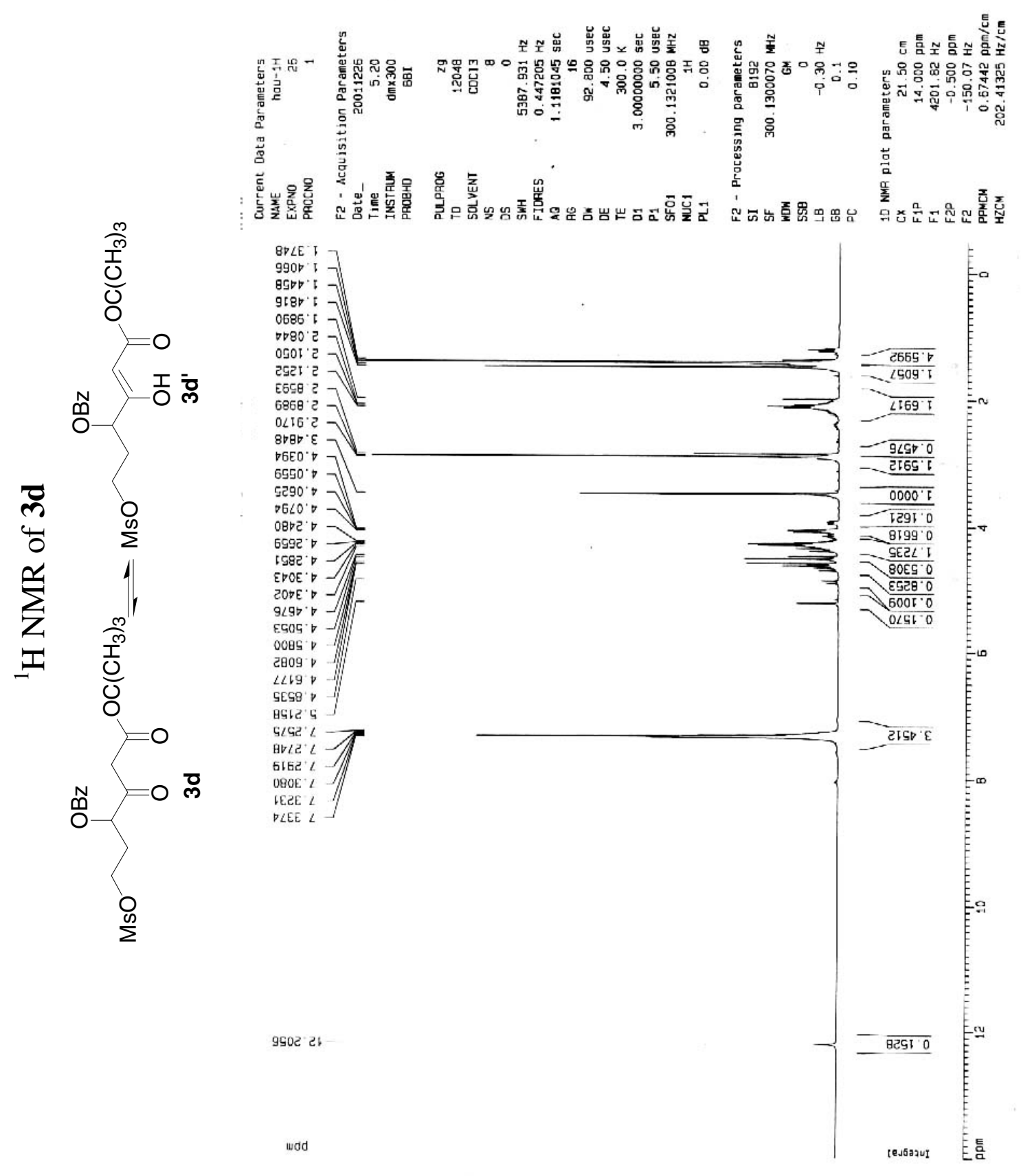

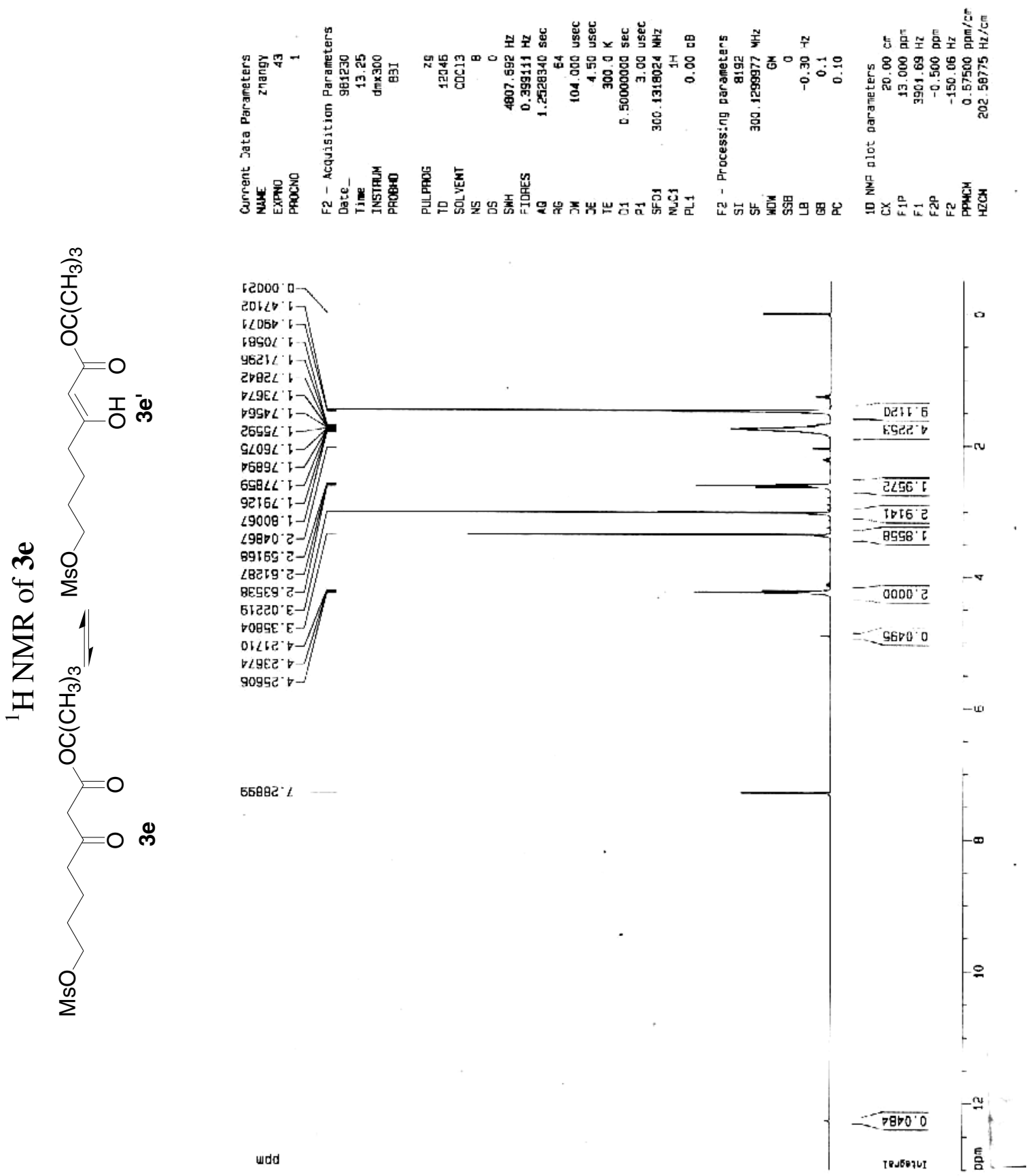

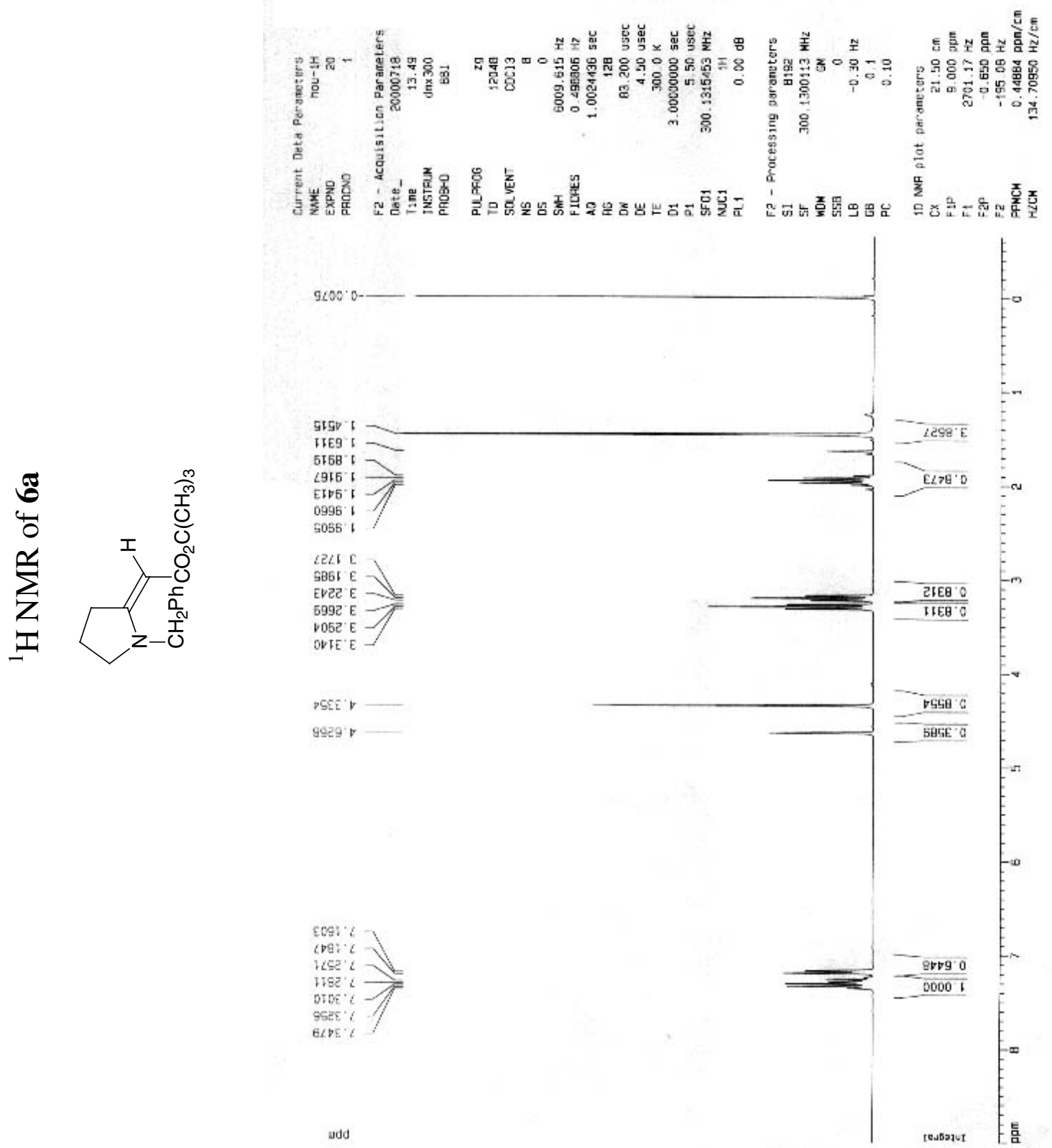

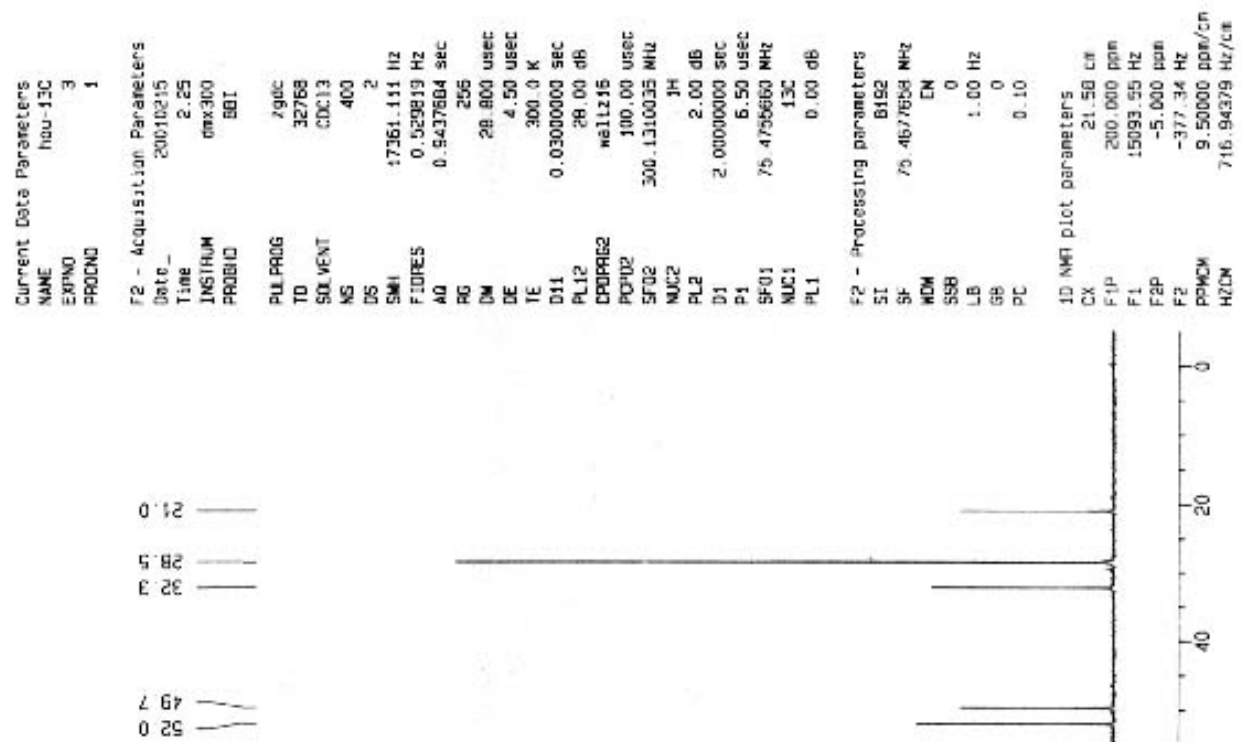

$\sqrt{6}$
$\sum_{0}^{4}$
$\sum_{0}^{0}$

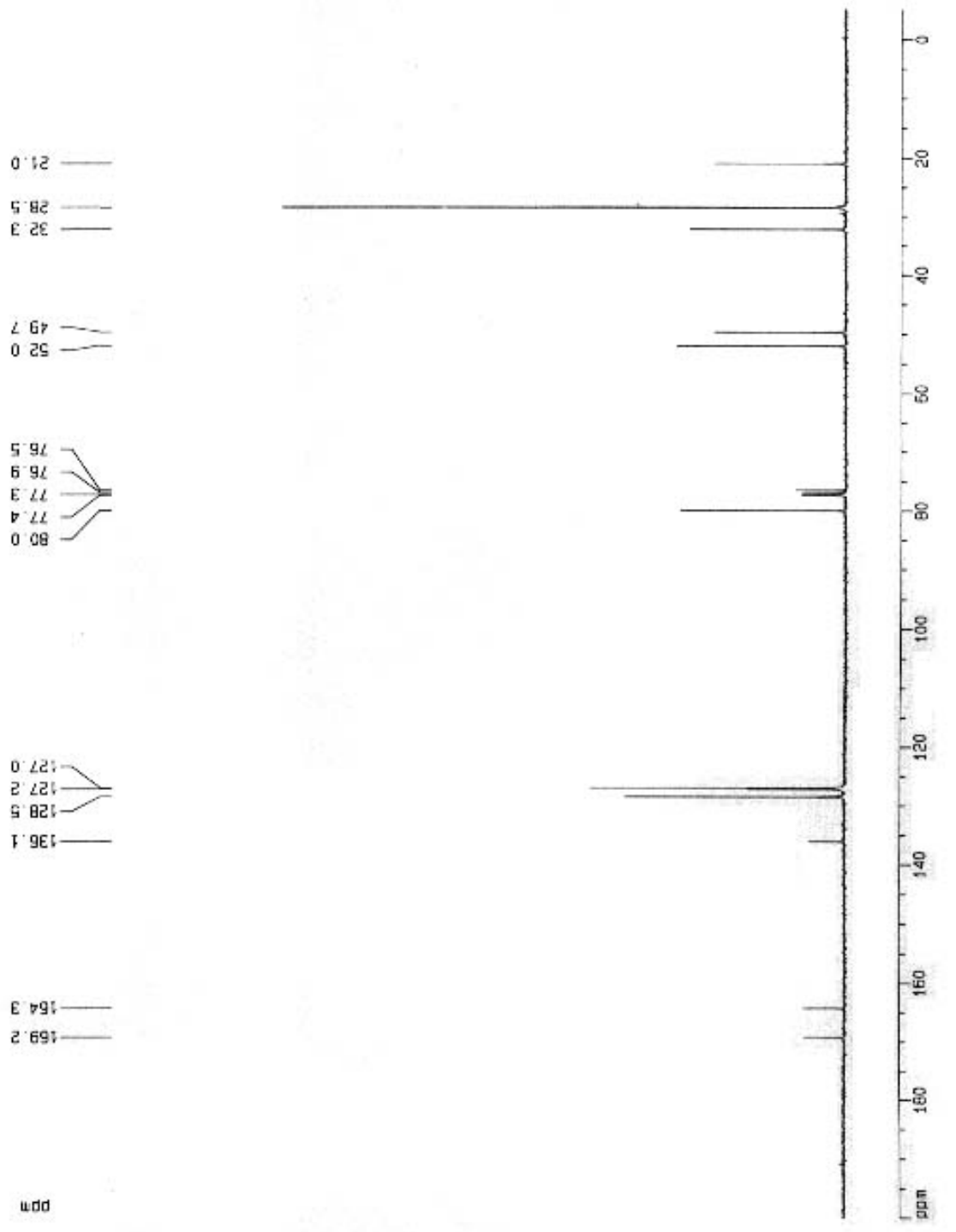




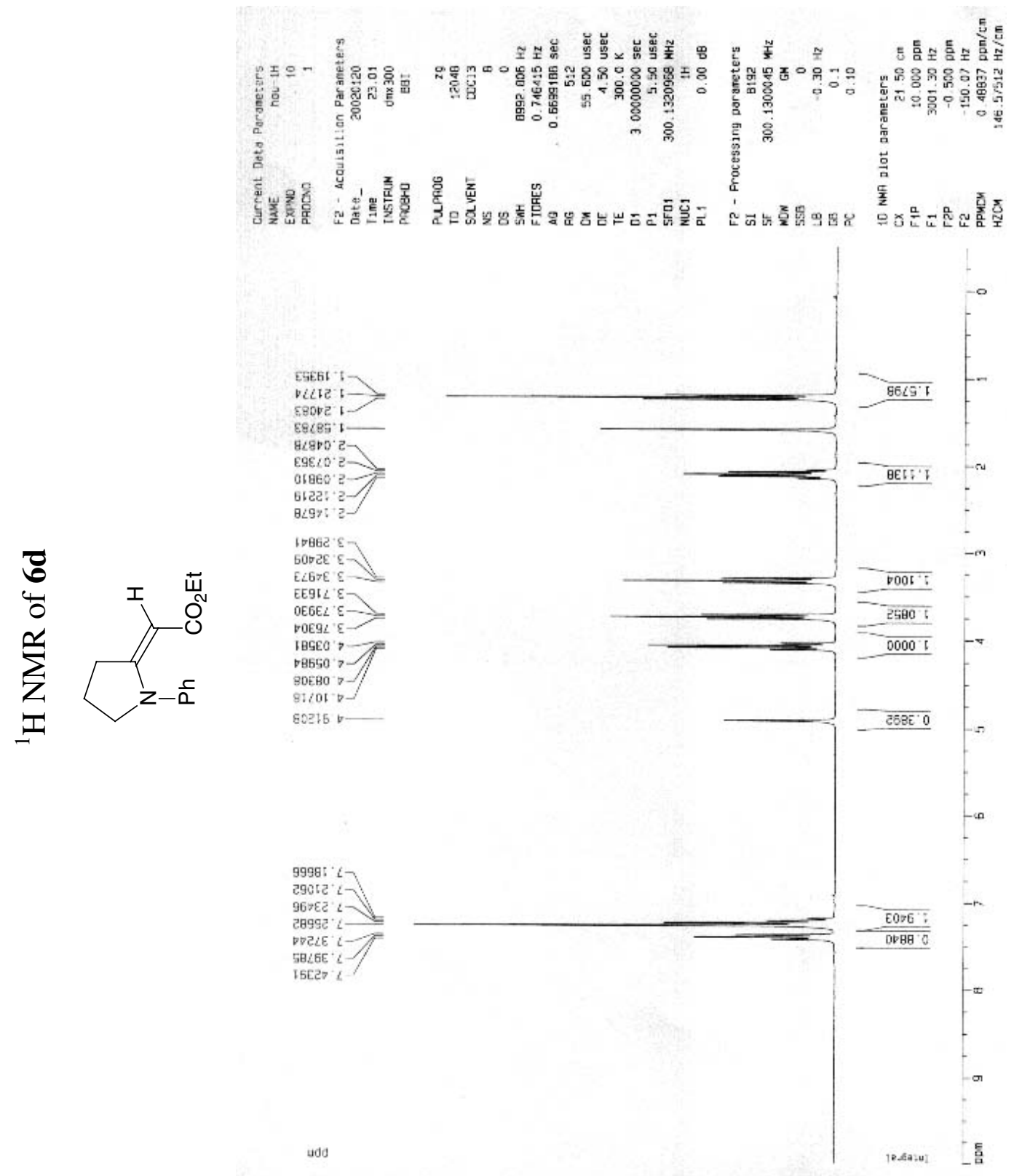




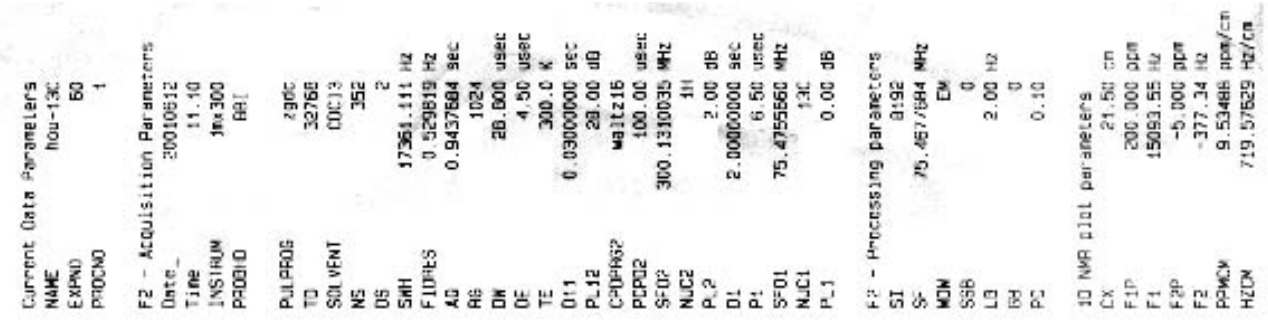

$\forall \forall$

E' 12

b' $2 E$

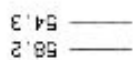

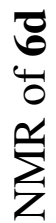

$7.9 L \longrightarrow$
$6.9 L \longrightarrow$

g. 08

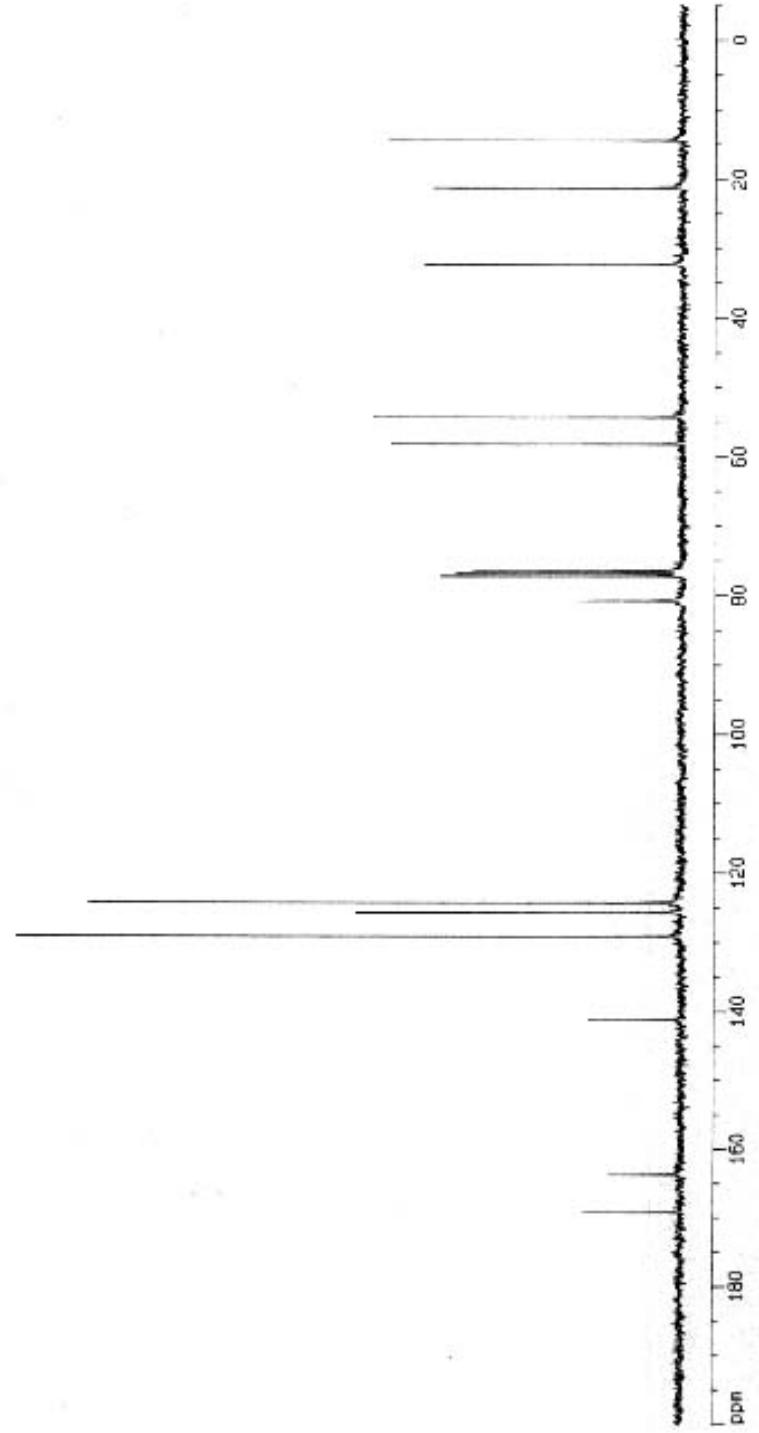

E.

$262:-$

2106

I. กิเ

$2 \cdot 696$

udo 

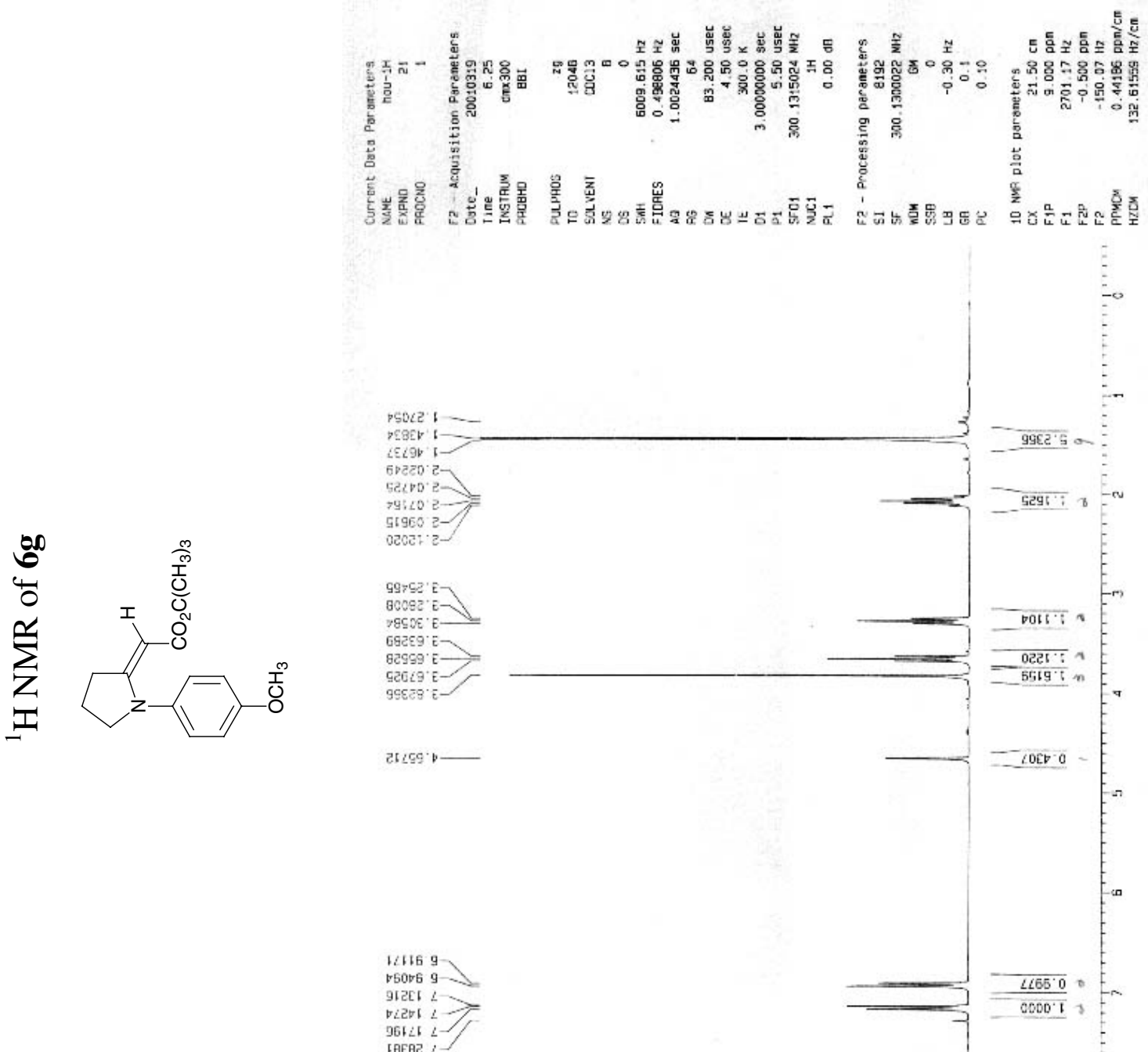

5 LEA2 $2-$

udd

PSOCE :-
PESEV :-

IE $\angle 9 \mathrm{~V}$ !

$6 D \angle 200^{\circ} 2$

$79: 20: 2-$

दा $19602-$

$\mathrm{Gg} \div \mathrm{CZ}, \mathrm{t}-$

gcosectin

69eद9 $\mathrm{t}-$

$82999^{\circ} \mathrm{c}-$

पct5 29 :

$25 G g \cdot b$
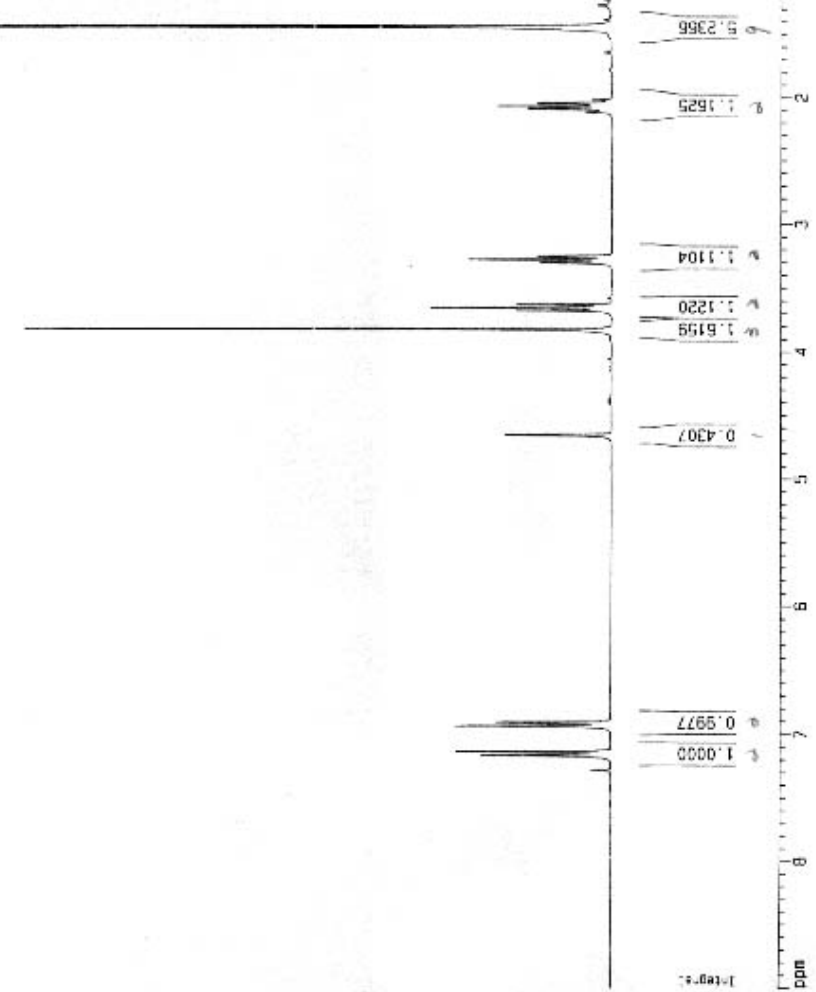


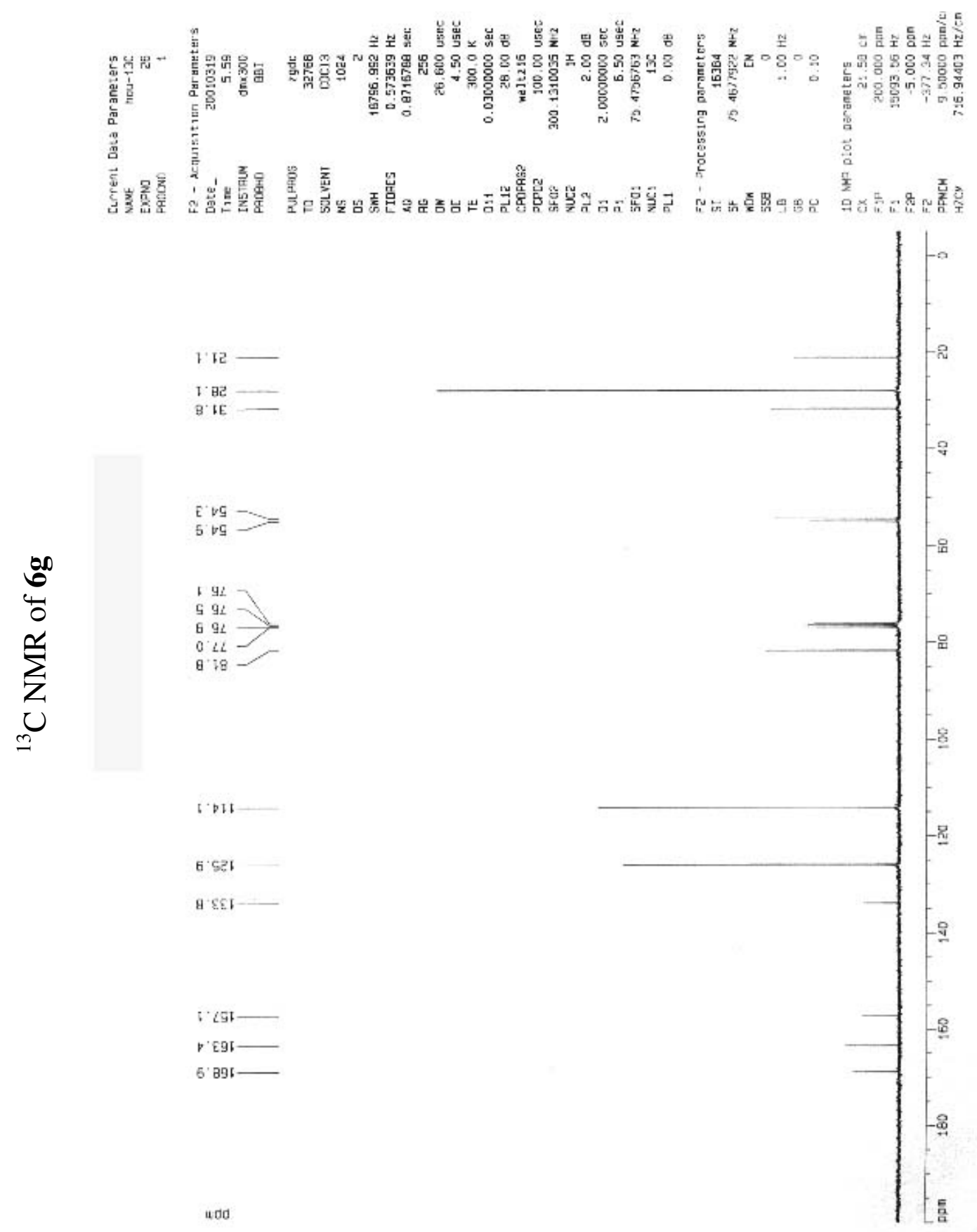



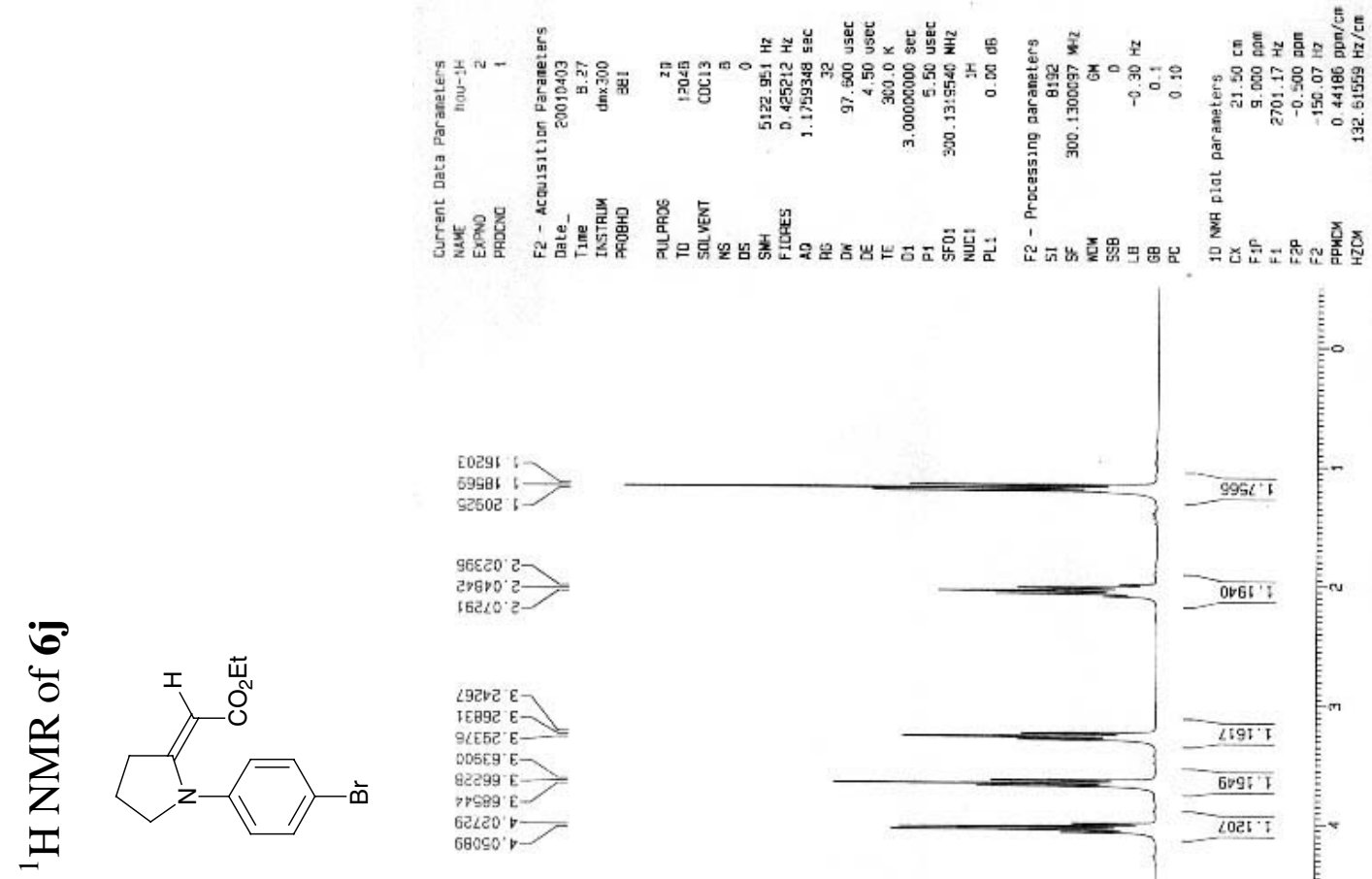

$00268>$

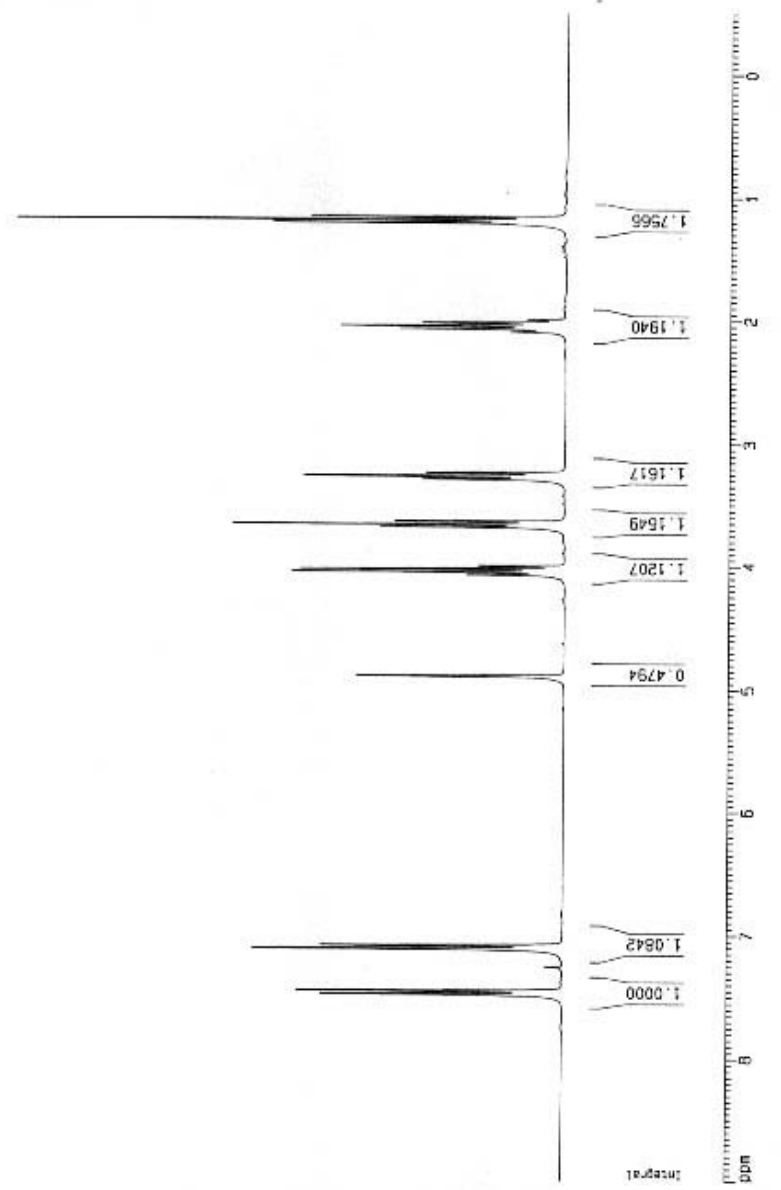




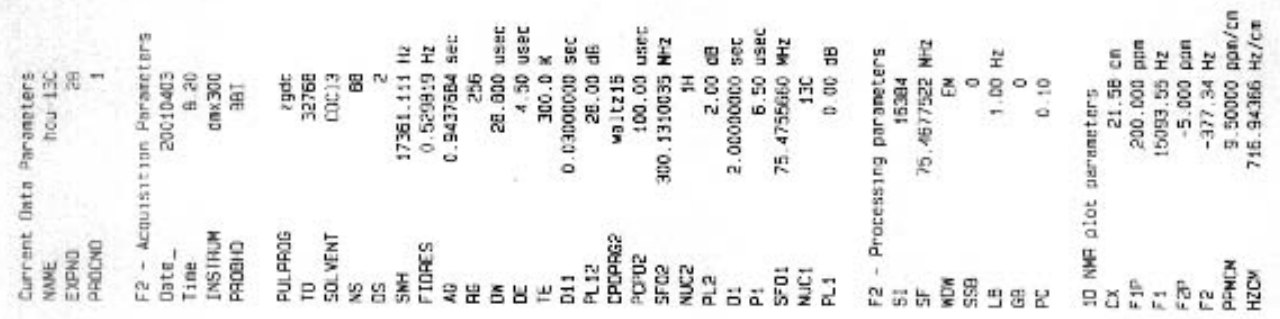

2.20

จ $己 \varepsilon$

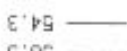

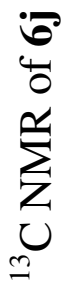

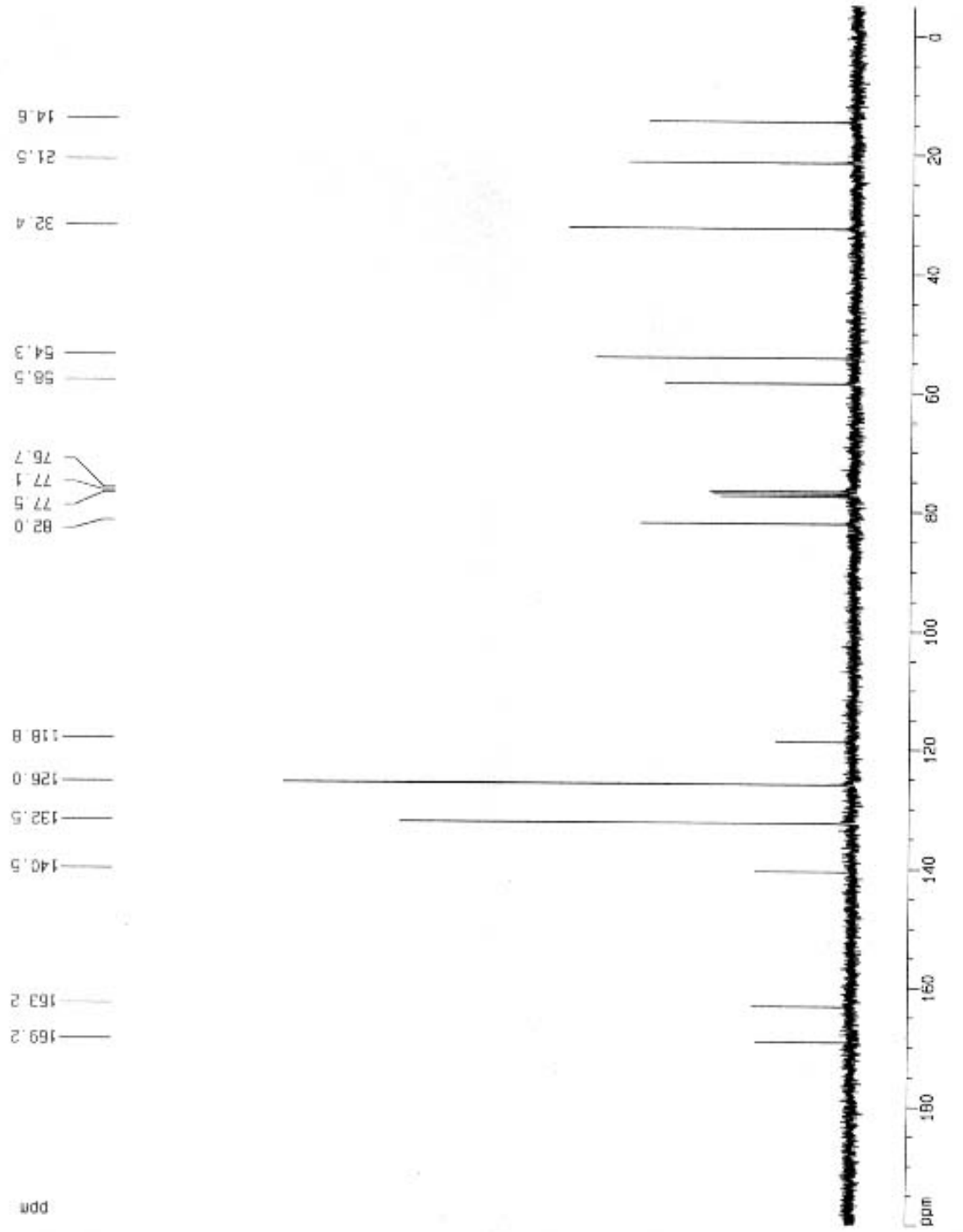



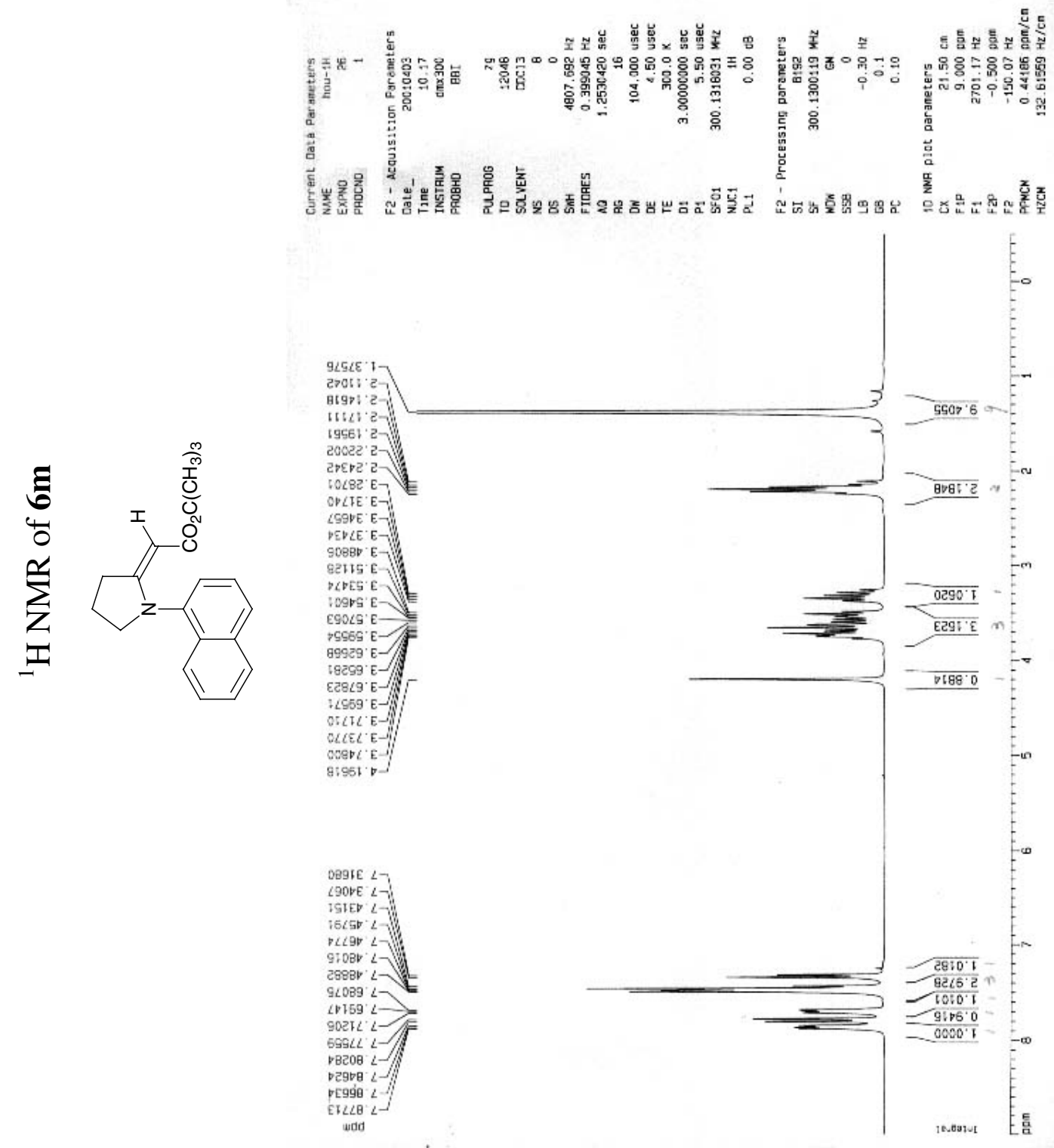

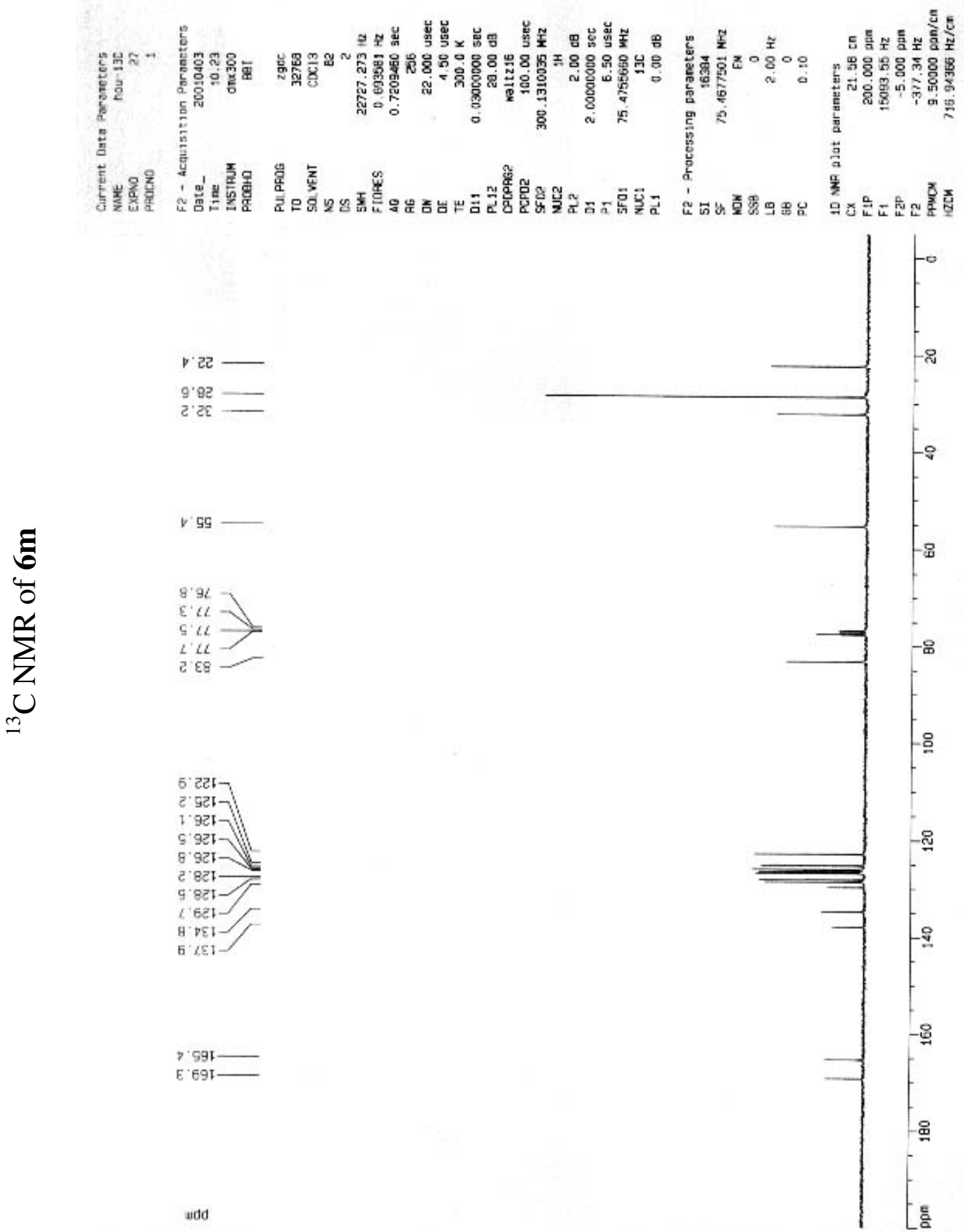

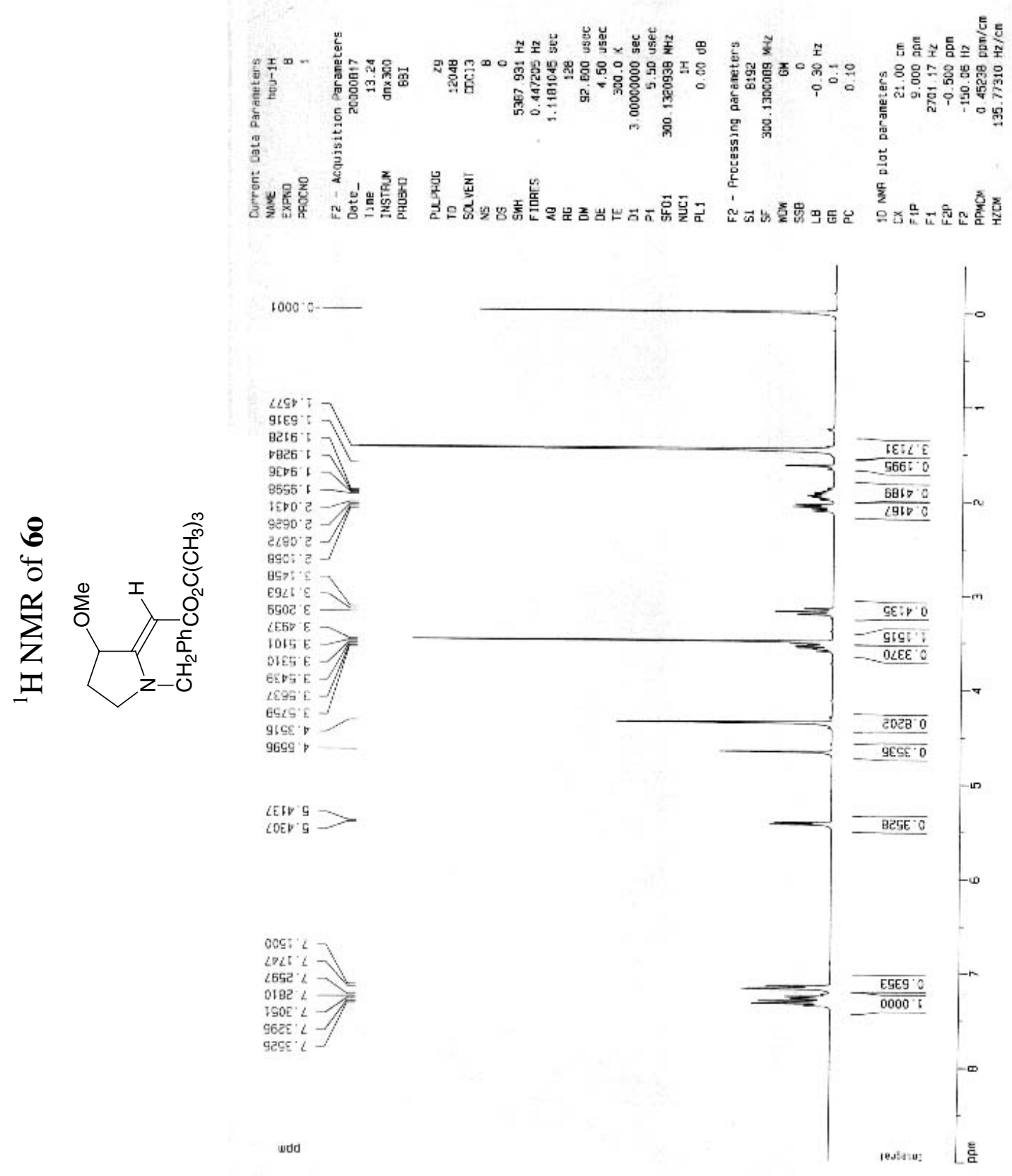

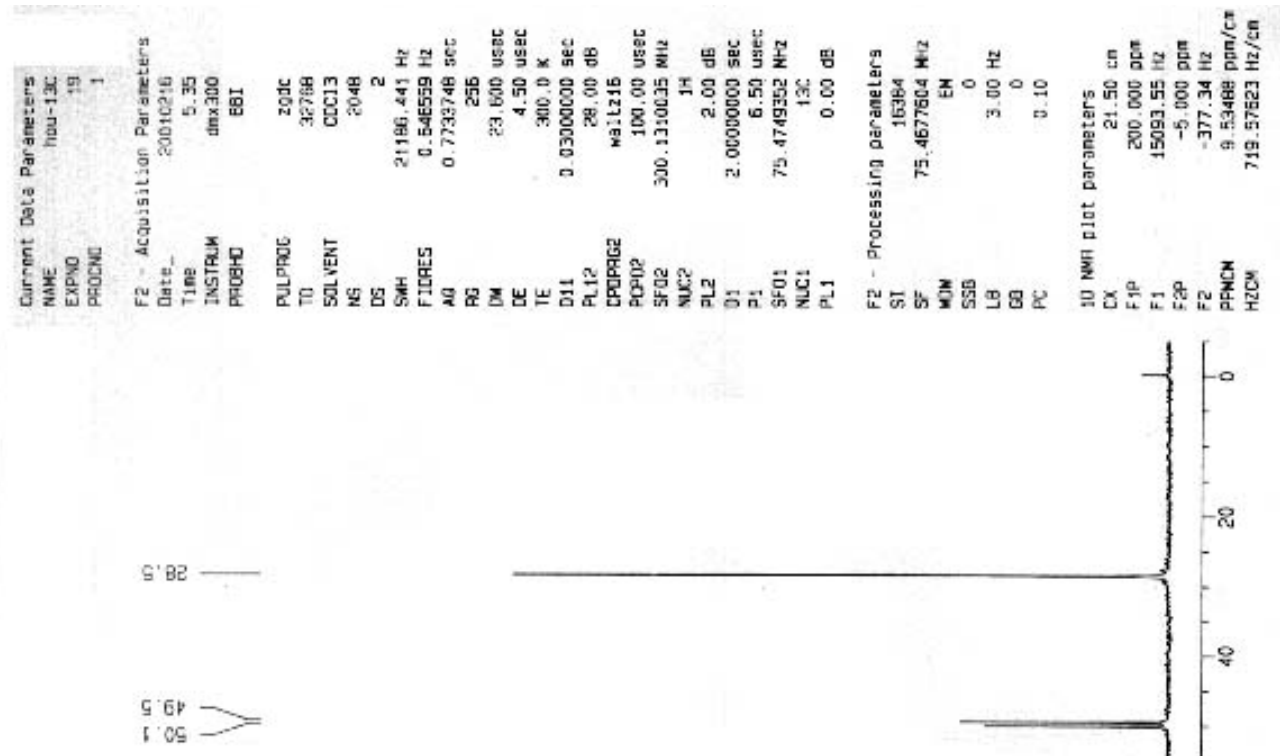

'. $Z \mathrm{~S}$

$\sum_{0}^{8}$

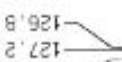

g.851-

$8 \cdot \operatorname{set}$
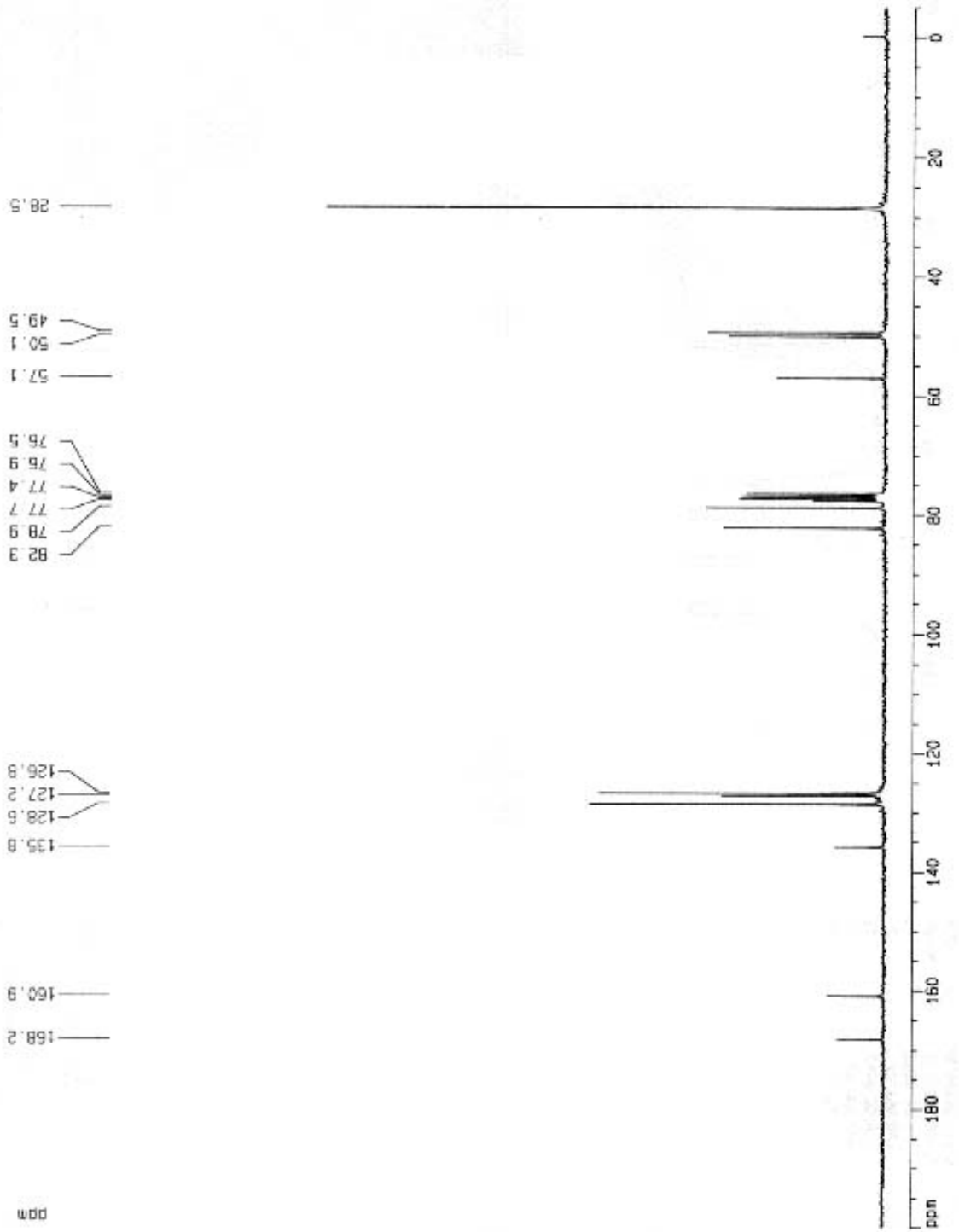


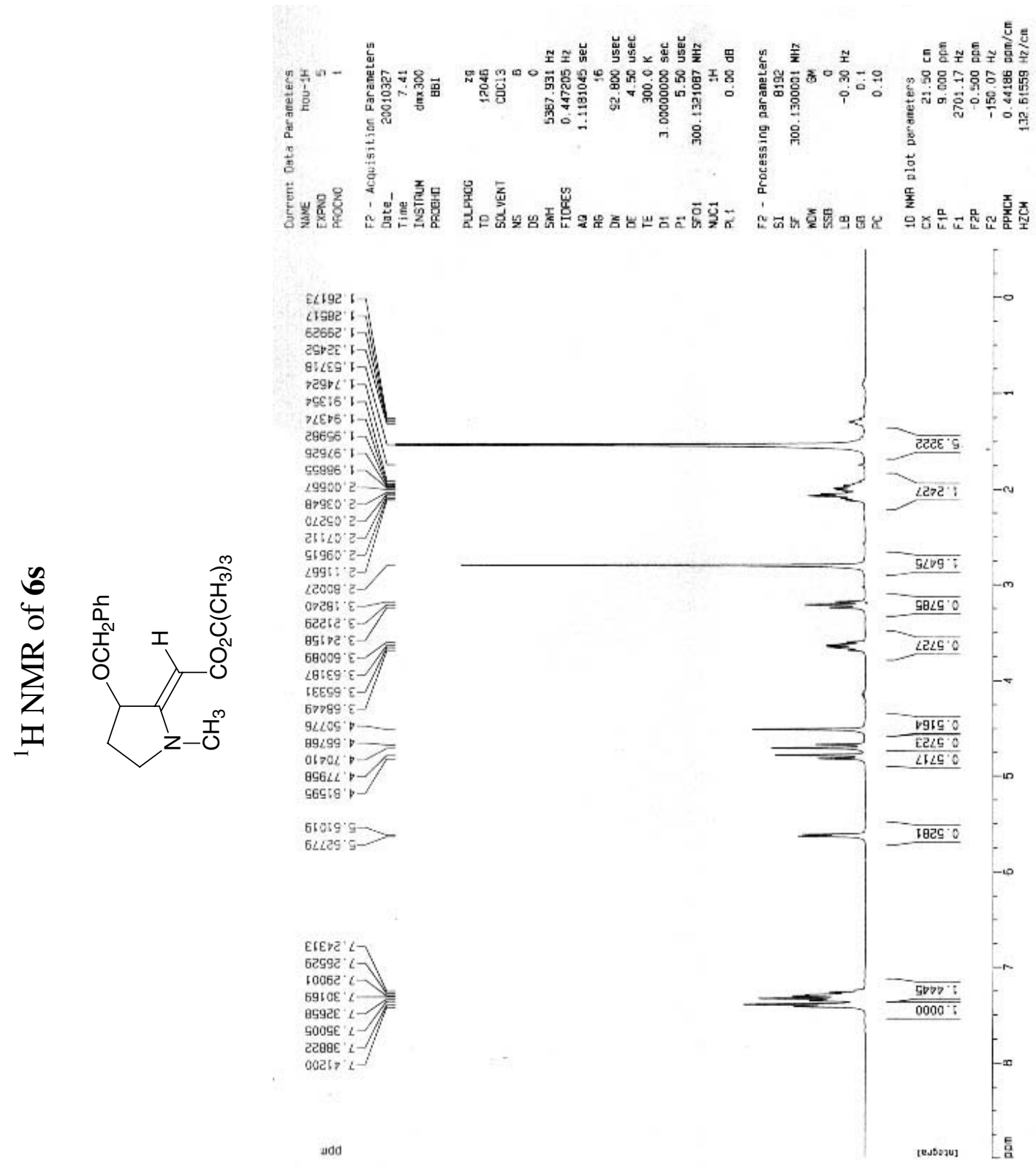



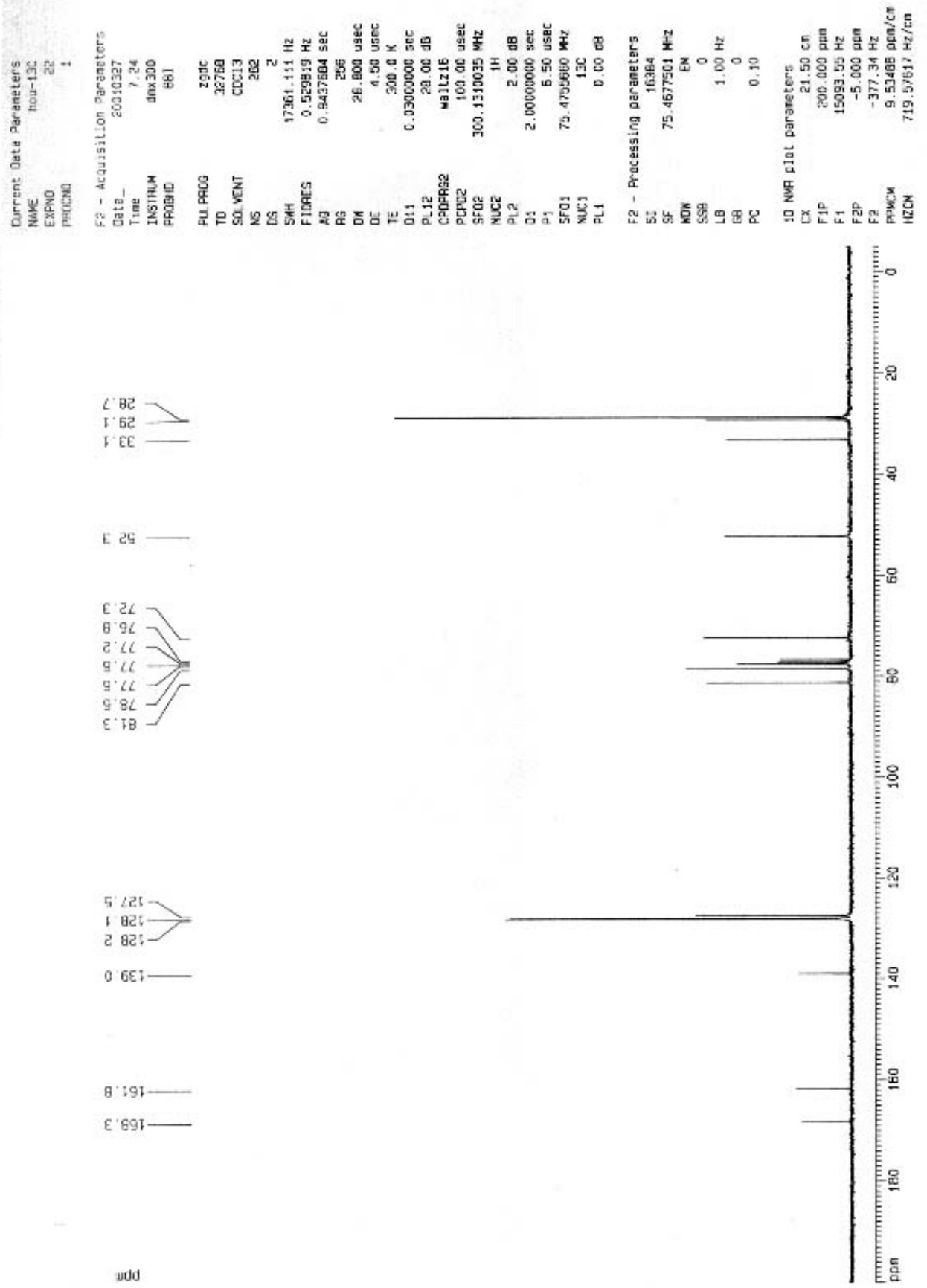

wdd

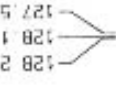

$06 \varepsilon$ -

8:191-

E' $691-$ 

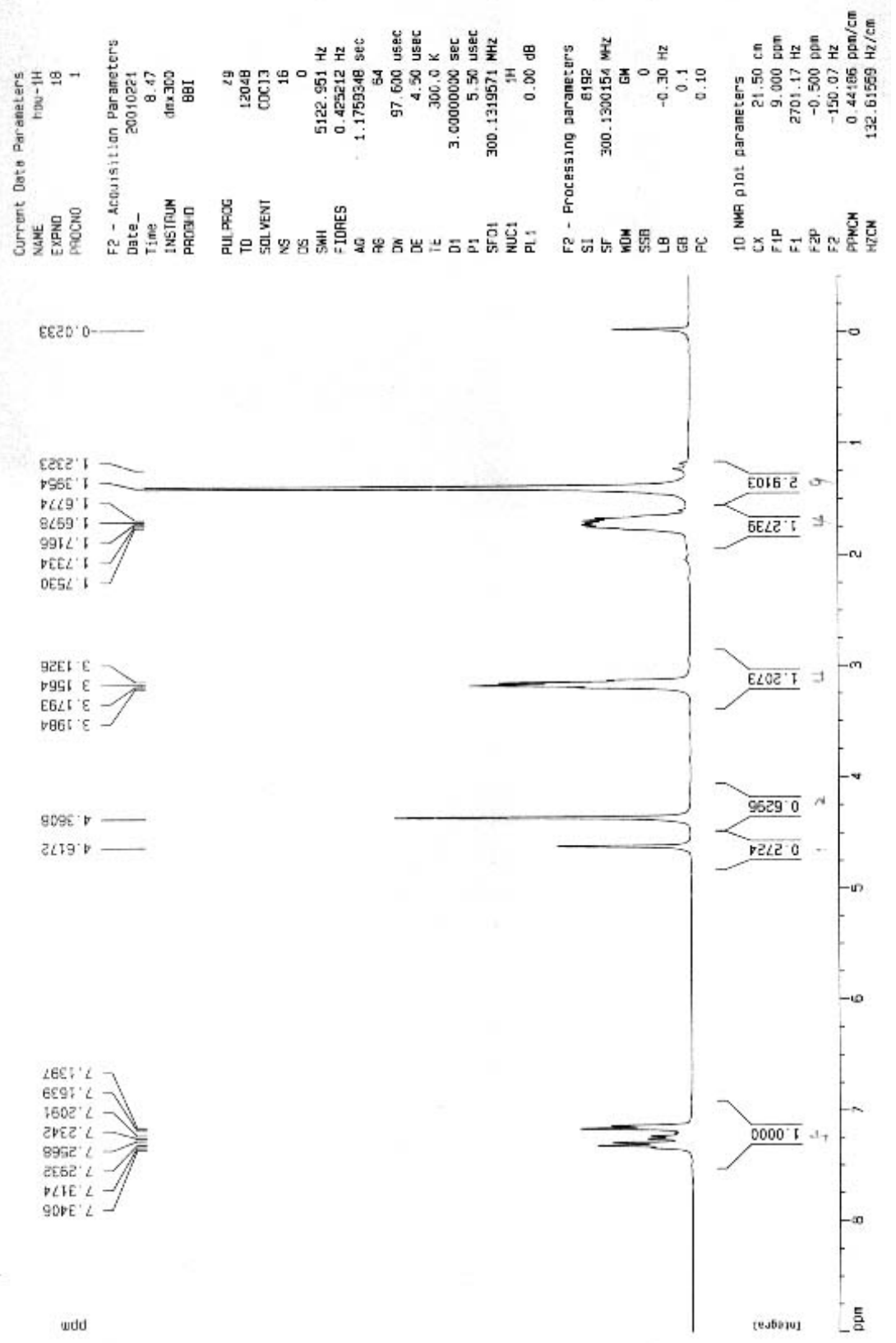

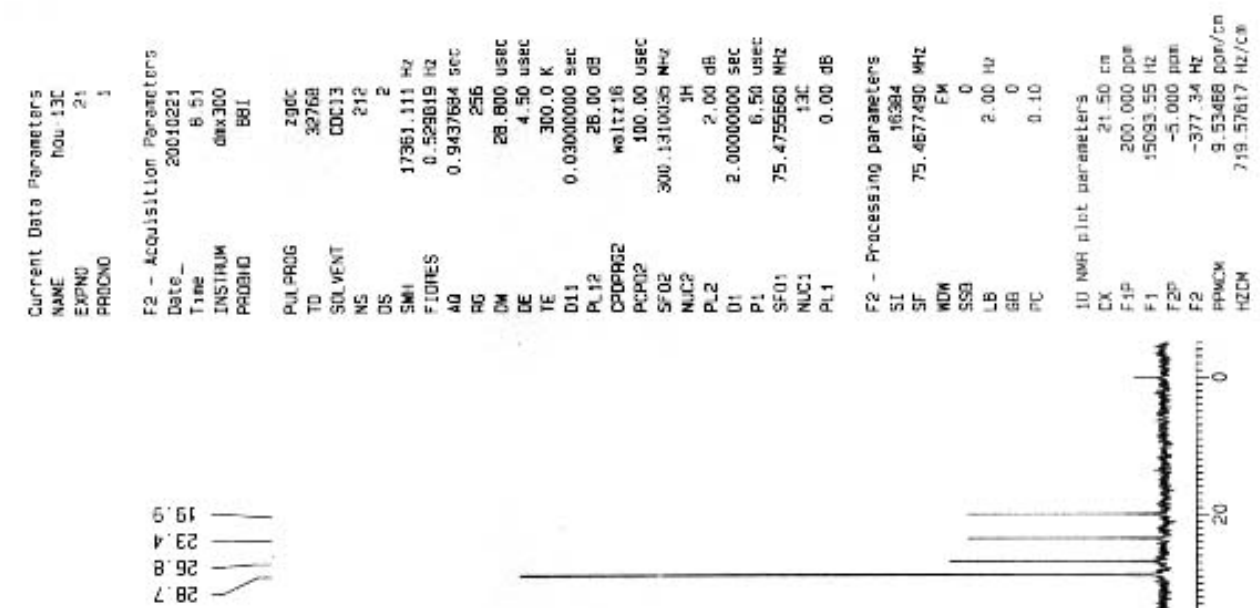

9. $6 \mathrm{p}$

0.59

5
0
0
0
0
0
0

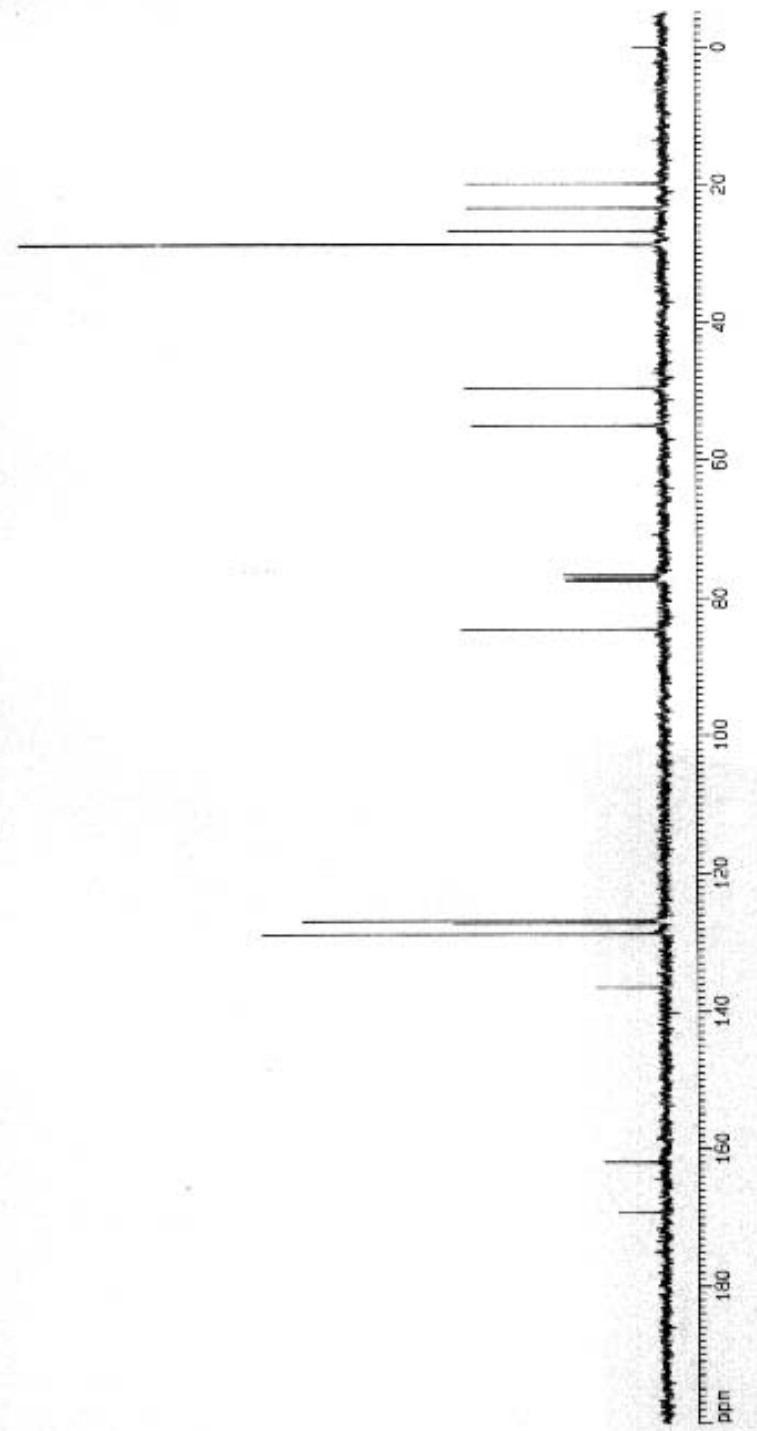



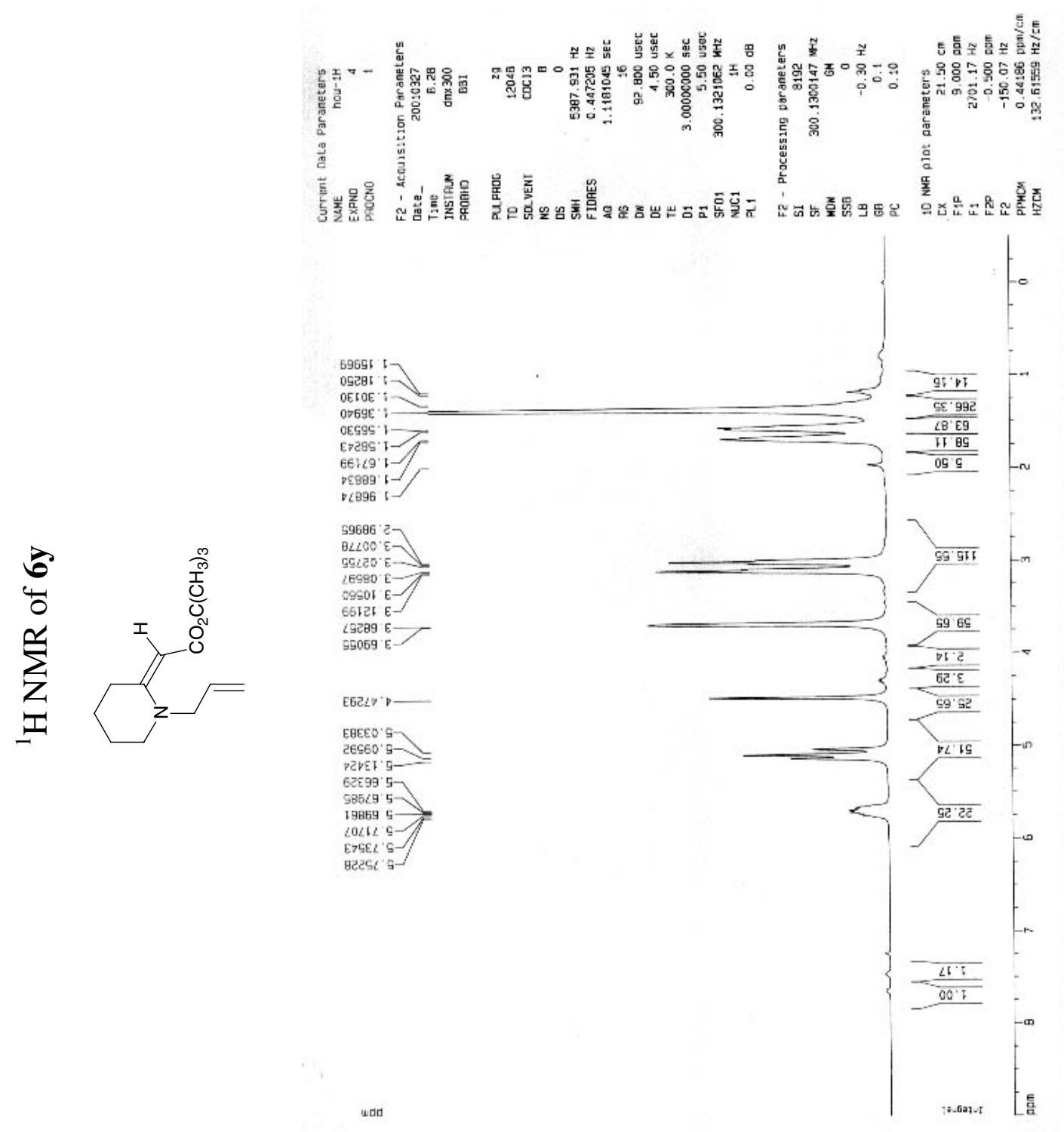

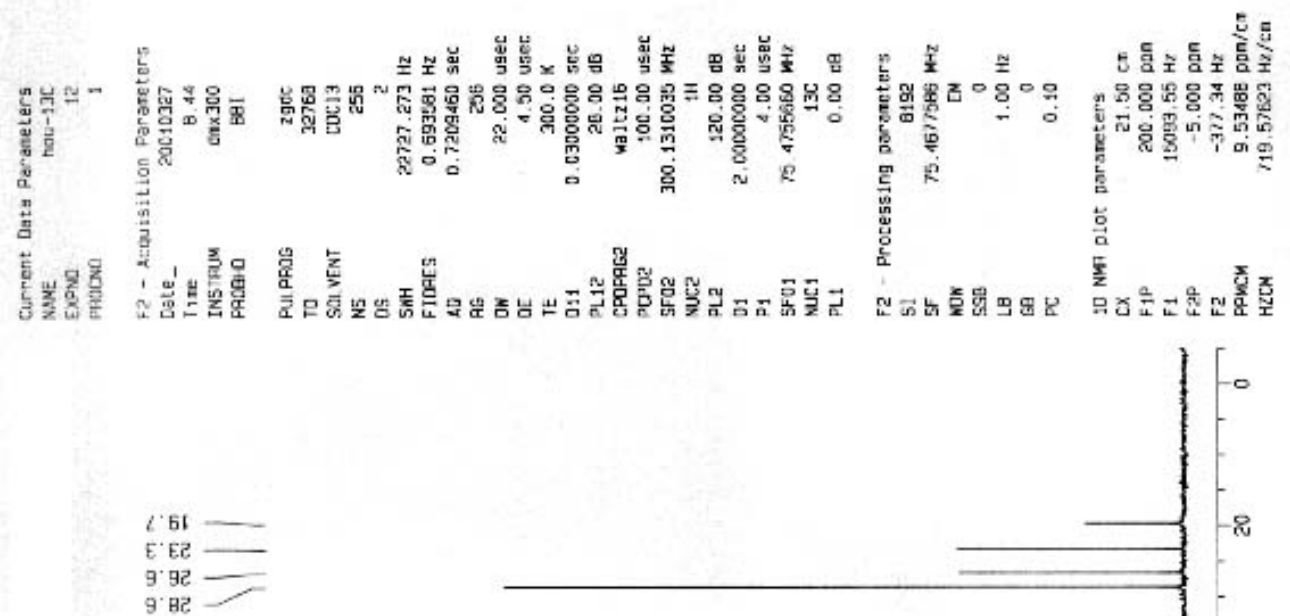

I.66 -

$2 \times 5$

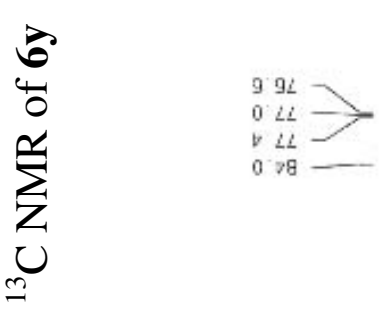

8. $9: 1$

: IEF-

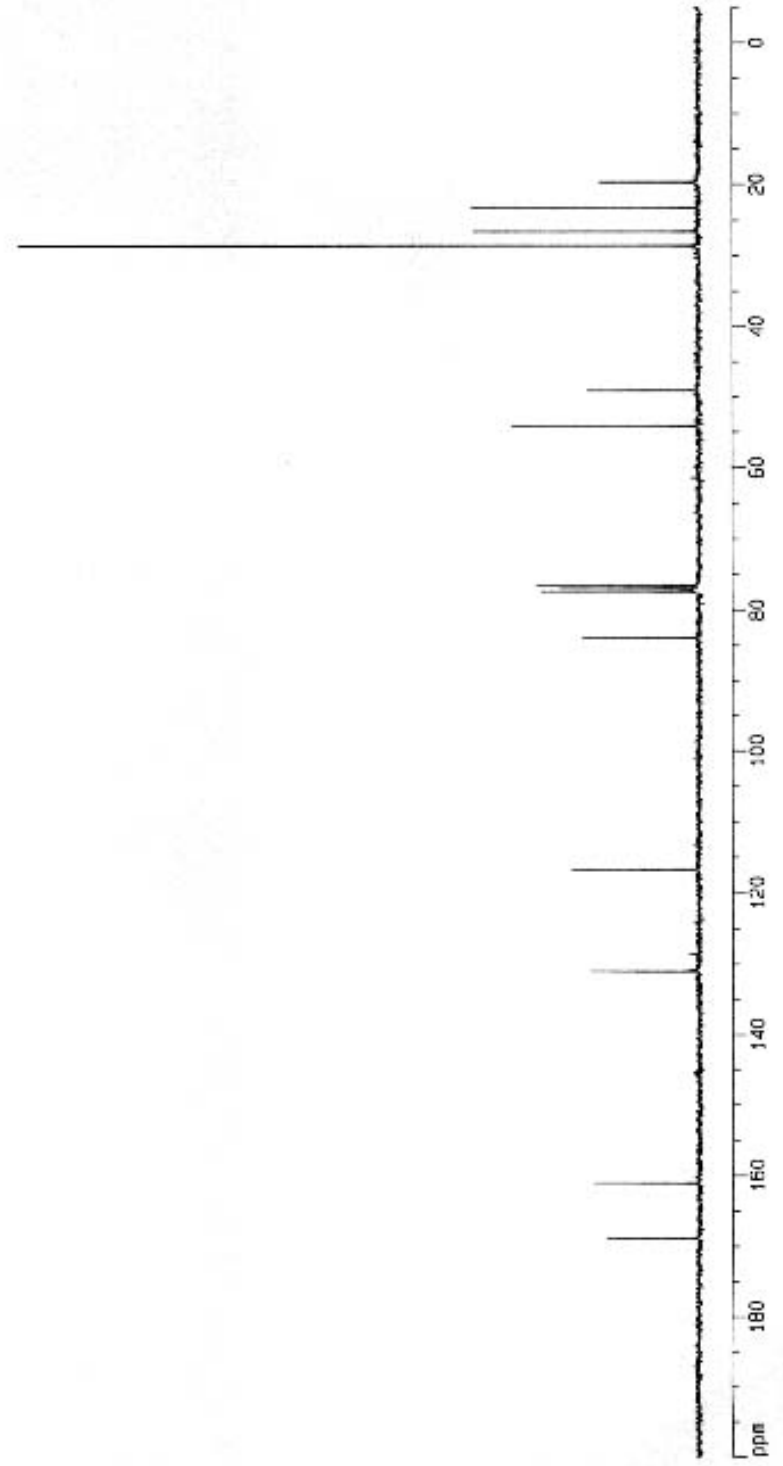

wod 